\title{
The radio structures of southern 2 -Jy radio sources: New ATCA and VLA radio images
}

\author{
R. Morganti ${ }^{1,2}$, T. Oosterloo ${ }^{2}$, C.N. Tadhunter ${ }^{3}$, R. Aiudi ${ }^{1}$, P. Jones ${ }^{4}$, and M. Villar-Martin ${ }^{5}$ \\ 1 Istituto di Radioastronomia, CNR, Via Gobetti 101, 40129 Bologna, Italy \\ 2 Netherlands Foundation for Research in Astronomy, Postbus 2, 7990 AA Dwingeloo, The Netherlands \\ 3 Dept. Physics, University of Sheffield, Sheffield S3 7RH, UK \\ ${ }^{4}$ Centre for Astronomy, University of Western Sydney, Nepean, PO Box 10, Kingswood, NSW 2747, Australia \\ ${ }^{5}$ Institute d'Astrophysique de Paris (IAP), 98 bis Bd. Arago, 75014 Paris, France
}

Received May 20; accepted September 21, 1999

\begin{abstract}
We present new radio images obtained with the Australia Telescope Compact Array (ATCA) and the Very Large Array (VLA) for a group of 14 galaxies belonging to the 2-Jy sample of radio sources. The new images improve the data already available on these objects and, in general, the database that we are building up on the sample. They will also be used for follow-up work where radio-optical comparison will be done.

We briefly discuss the core dominance parameter $(R)$ for the objects for which the new data have given new information and, in particular, for broad line radio galaxies (BLRG). One of the BLRG does not show a core, even at $3 \mathrm{~cm}$, and this is at variance with the general tendency of BLRG to have relatively strong cores. The depolarization is also discussed for a group of small double-lobed radio galaxies.
\end{abstract}

Key words: galaxies: active — radio continuum: galaxies - polarisation

\section{Introduction}

Multiwaveband studies of Active Galactic Nuclei (AGN) have become an essential part in understanding these objects. Indeed, our knowledge of this group of objects is not complete until the relation between their (e.g.) optical, radio and X-ray emission is clear. Moreover, part of the study of AGN concentrates on the importance of "unified schemes" in explaining the appearance of these objects. Tests for these schemes are more efficient when different characteristics in different wavebands have to be explained.

Send offprint requests to: R. Morganti,

e-mail: morganti@nfra.nl
For these reasons we started a few years ago the study of a complete sample of radio sources with the idea of building an homogeneous database that could allow a comprehensive investigation of various aspects and open questions connected with AGNs. For this study we have used the southern part of the so-called 2-Jy sample (Wall \& Peacock 1985) with selection criteria $S_{2.7 \mathrm{GHz}}>2 \mathrm{Jy}$, $\delta<+10^{\circ}$ and $z<0.7$. This sample includes 88 radio sources (radio galaxies and quasars) of many different classes, i.e. Fanaroff-Riley (Fanaroff \& Riley 1974) types I and II, core/halo, Compact Steep Spectrum (CSS) and flat spectrum sources. For all these objects we have now collected optical spectra and polarimetry (Tadhunter et al. 1997; Tadhunter et al. 1998), X-ray data (Siebert et al. 1996; Padovani et al. 1999; Trussoni et al. 1999) and radio data. The latter comprise images (VLA and ATCA, Morganti et al. 1993) and high resolution core flux density measurements (Morganti et al. 1997a).

A number of interesting results have been already obtained from this database (see Morganti \& Tadhunter 1997; Morganti et al. 1997a; Tadhunter et al. 1997; Tadhunter et al. 1998 and ref. therein). However, together with the results some new questions have arisen which would benefit from higher quality data than available at present. In particular, for a number of sources the radio maps available are not good enough for a proper morphological classification and to derive other important parameters.

In order to improve the available radio images we have carried out new observations, for a number of radio galaxies in the sample, using either the VLA or the ATCA, according to the declination of the sources. This work can be, therefore, considered as a follow-up and completion of the radio study of this sample presented in Morganti et al. (1993). In this paper we present the new total intensity and polarisation radio images obtained for 14 radio 


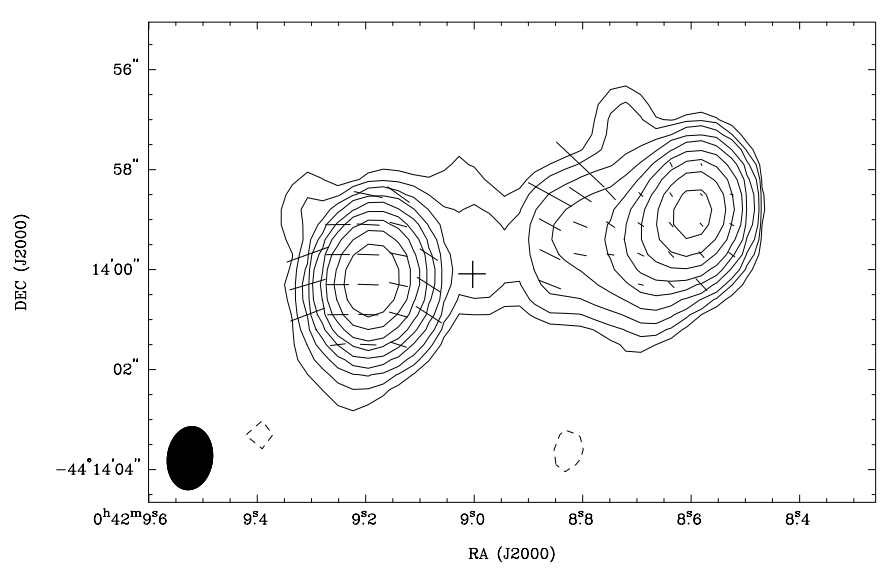

Fig. 1. Image of 0039-44 at 3-cm (full resolution) with superimposed vectors indicating the projected electric field direction. The vectors are proportional in length to the fractional polarisation $(1 \operatorname{arcsec}=0.26$ ratio). The contour levels are: $0.5 \times-1$, $1,2,4,8,16,32,64,128,256 \mathrm{mJy}_{\text {beam }}{ }^{-1}$. The peak flux is 289.0 mJy beam $^{-1}$. The synthesized beam of the observations is marked in the low left corner. The cross indicates the position (from di Serego et al. 1994) of the optical galaxy

galaxies. The objects selected are very different in morphology: among them there are both small double-lobed and large objects that needed a better radio image for a proper classification. These images will be mainly used for follow up work where radio-optical comparison will be done.

Throughout this paper we assume that $H_{0}=$ $50 \mathrm{~km} \mathrm{~s}^{-1} \mathrm{Mpc}^{-1}$ and $q_{0}=0$.

\section{Observations}

We have observed 14 radio galaxies with either the Australia Telescope Compact Array (ATCA) ${ }^{1}$ or the Very Large Array (VLA) ${ }^{2}$ depending on their declination. One exception is 1938-15, which was observed with both the instruments. The general characteristics of the observed objects are listed in Table 1. The total power, calculated at $4.8 \mathrm{GHz}$, and the spectral index, estimated between 2.7 and $4.8 \mathrm{GHz}\left(\alpha_{2.3 \mathrm{GHz}}^{4.8 \mathrm{GH}}\right.$, defined as $\left.S \propto \nu^{-\alpha}\right)$, have been taken from Wall \& Peacock (1985).

Large angular (LAS) and linear (LLS) sizes (taken from the lower resolution images available in literature, see notes to the sources) and measured from the lower contour, are also listed.

\footnotetext{
1 Operated by the CSIRO Australia Telescope National Facility.

2 The National Radio Astronomy Observatory is operated by Associated Universities, Inc., under contract with National Science Foundation.
}

\subsection{ATCA observations}

Seven objects in the most southern part of this sample $\left(\delta<-30^{\circ}\right.$, with the exception of $\left.1938-15\right)$ were observed with the ATCA. These observations were done on the 4-7 April 96 in the $6 \mathrm{~km}(6 \mathrm{~A})$ configuration and on the 8 May 96 in the $1.5 \mathrm{~km}(1.5 \mathrm{D})$ configuration. Data were taken simultaneously at $4.8 \mathrm{GHz}(6 \mathrm{~cm})$ and $8.6 \mathrm{GHz}(3 \mathrm{~cm})$, each with a bandwidth of $128 \mathrm{MHz}$.

The $3 \mathrm{~cm}$ observations (with the $6 \mathrm{~km}$ configuration) are essential to improve the previously available radio images and to be able to detect, e.g., jet structures (if any) close to the core. However, for the three larger sources (0043-42, 0625-53 and 2058-28) we have also obtained observations with the $1.5 \mathrm{~km}$ configuration in order to better image the extended emission. The data from the two configurations have been combined to obtain the best images. For two objects (0043-42 and 2058-28) the resolution of our combined $3 \mathrm{~cm}$ data was still too high to properly image the extended emission. Only the $6 \mathrm{~cm}$ data will be presented here for 2058-28, while for $0043-42$ we only show an image of the hot-spots at $3 \mathrm{~cm}$.

Each source was observed in scans of about 20 min for a total of about $6 \mathrm{~h}$ in each configuration. The scans were spread throughout a 12 hour observing period in order to optimise the $(u, v)$ coverage within the available integration time. The ATCA observing parameters are summarised in Table 2.

The ATCA data were calibrated by using the MIRIAD package (Sault et al. 1995), which is necessary for the polarisation calibration of ATCA data. The flux density scale was calibrated against observations of PKS 1934-638, assumed to be $5.83 \mathrm{Jy}$ at $4.8 \mathrm{GHz}$ and $2.84 \mathrm{Jy}$ at $8.6 \mathrm{GHz}$ according to the latest analysis by Reynolds (1996). A few iterations of phaseonly self-calibration were then applied to each source. Together with the total intensity images we have also obtained the images for the Stokes parameters $(Q, U)$, the polarised intensity $\left(P=\left(Q^{2}+U^{2}\right)^{1 / 2}\right)$ and position-angle $(\chi=0.5 \arctan (U / Q))$ images. The polarised intensity and the fractional polarisation $(m=P / I)$ were estimated only for the pixels for which $P>3 \sigma_{Q U}$.

In order to compare the $8-\mathrm{GHz}$ and the $5-\mathrm{GHz}$ data (being taken with the same array they have different resolution), we have generated 8- and 5-GHz Stokes $I, Q$ and $U$ images at the best 5 - $\mathrm{GHz}$ resolution (see Table 2). This was done by convolving the $8-\mathrm{GHz}$ visibilities with the appropriate Gaussian during imaging, thus weighting down the longer spacing. The derived depolarization is defined as the $D P_{3}^{6}=m_{6 \mathrm{~cm}} / m_{3} \mathrm{~cm}$. We also used the position angles at the two frequencies to derive the twopoint Faraday rotation $(R M)$. The $R M$ is defined as $\chi\left(\lambda^{2}\right)=\chi_{\text {intr }}+R M \lambda^{2}$ where $\chi_{\text {intr }}$ is the intrinsic position angle and $\chi$ the apparent position angle at the $\lambda$ of the observations. Since the position angles are ambiguous by $n \pi$, an ambiguity affects also the $R M$ calculated from 
Table 1. General characteristics

\begin{tabular}{lcccccc}
\hline \hline Object & & $z$ & $\alpha_{2.3 \mathrm{GHz}}^{4.8 \mathrm{GHz}}$ & $\begin{array}{c}\log P_{\text {tot }}^{6 \mathrm{~cm}} \\
\mathrm{~W} \mathrm{~Hz}^{-1}\end{array}$ & $\begin{array}{c}\text { LAS } \\
\operatorname{arcsec}\end{array}$ & $\begin{array}{c}\text { LLS } \\
\mathrm{kpc}\end{array}$ \\
\hline & & & & & & \\
$0034-01$ & 3C 15 & 0.073 & 0.79 & 25.59 & 42 & 80 \\
$0035-02$ & 3C 17 & 0.220 & 0.72 & 26.81 & 38 & 181 \\
$0039-44$ & & 0.346 & 0.93 & 26.93 & 9.5 & 62 \\
$0043-42$ & & 0.116 & 0.87 & 26.28 & 160 & 460 \\
$0117-15$ & \multirow{2}{*}{ 3C 38 } & 0.565 & 0.90 & 27.54 & 14 & 120 \\
$0409-75$ & & 0.693 & 0.86 & 28.18 & 9 & 85 \\
$0442-28$ & OF-271 & 0.147 & 0.93 & 26.38 & 105 & 367 \\
$0453-20$ & OF-289 & 0.035 & 0.73 & 25.00 & 27 & 26 \\
$0625-53$ & & 0.054 & 1.17 & 25.40 & 90 & 130 \\
$1602+01$ & \multirow{2}{*}{ C 327.1 } & 0.462 & 1.07 & 27.21 & 19 & 147 \\
$1938-15$ & OV-164 & 0.452 & 0.82 & 27.47 & 6.5 & 50 \\
$1954-55$ & & 0.060 & 0.78 & 25.58 & 360 & 576 \\
$2058-28$ & & 0.038 & 0.74 & 25.11 & 600 & 628 \\
$2314+03$ & \multirow{2}{*}{ 3C 459 } & 0.220 & 0.97 & 26.54 & 10 & 48 \\
\hline \hline
\end{tabular}
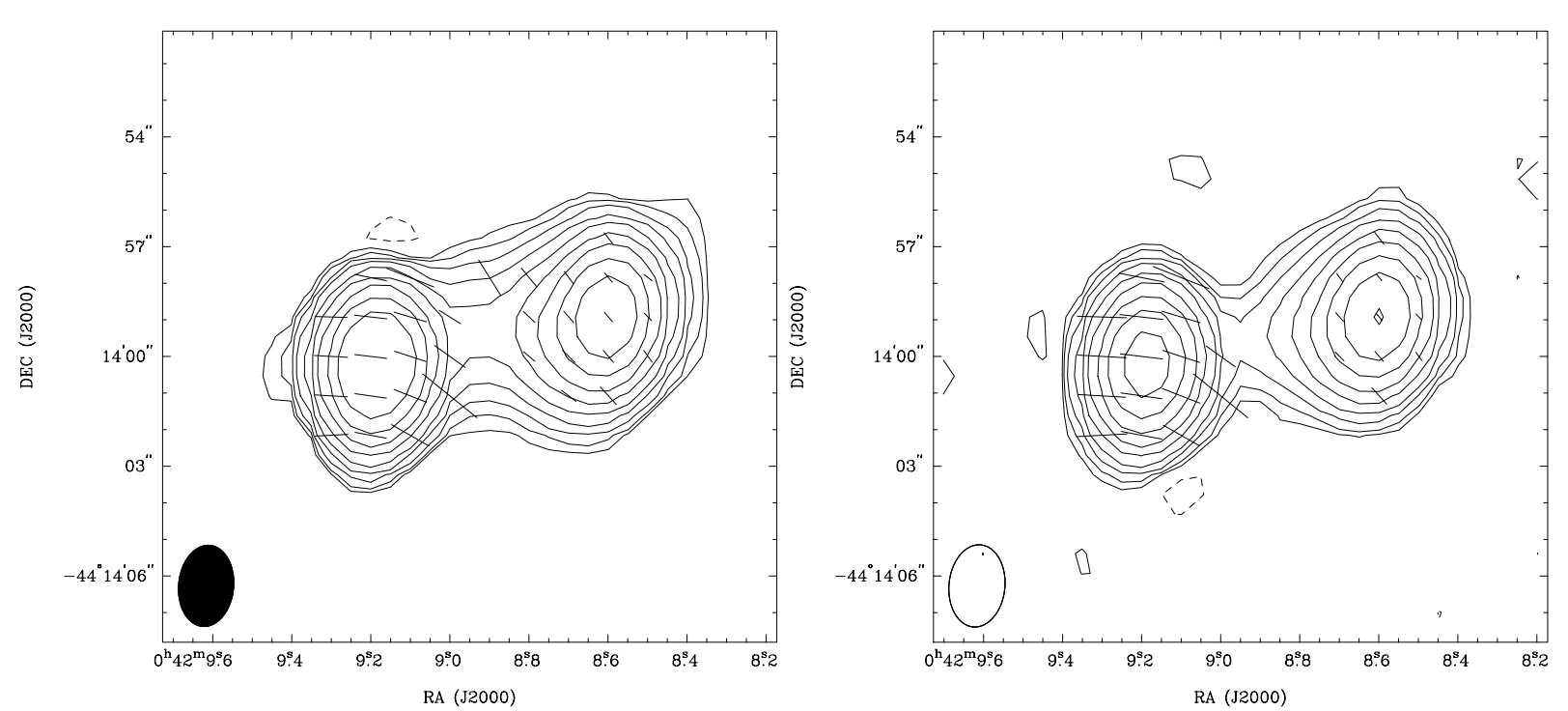

Fig. 2. Image of 0039-44 at 6-cm (Left) and 3-cm (Right) lower resolution with superimposed vectors indicating the projected electric field direction. The vectors are proportional in length to the fractional polarisation $(1 \operatorname{arcsec}=0.08 \mathrm{ratio}$ for the $6 \mathrm{~cm})$. The contour levels are: $0.8 \times-1,1,2,4,8,16,32,64,128,256 \mathrm{mJy}^{\text {beam }}{ }^{-1}$. The peak flux at $6 \mathrm{~cm}^{\text {is }} 587.8 \mathrm{mJy} \mathrm{beam}^{-1}$ and at $3 \mathrm{~cm}$ is $305.0 \mathrm{mJy}^{\text {beam }^{-1}}$

only two frequencies. Thus, the $R M$ presented here will have to be confirmed by observing the objects at at least one other frequency.

\subsection{VLA observations}

VLA snapshot observations were obtained for 8 sources in the declination range north of $-30^{\circ}$ using the A-array configuration on the 26 Oct. 96 . The VLA observations are summarised in Table 3 . The observations were carried out using the standard $6 \mathrm{~cm}(4.8 \mathrm{GHz})$ continuum mode, that is, with two $50 \mathrm{MHz}$-wide channels at bandwidth- separated frequencies $(4.835 \& 4.885 \mathrm{GHz})$. Each source was observed in two scans of about 15 minutes.

Data reduction followed standard procedures using the NRAO software package AIPS. The flux scale was calibrated against observations of the flux standards 3C 48 \& 3C 286, adopting the standard scaling of Baars (1977). A few iterations of phase-only self-calibration were applied to each source. Again, together with the total intensity images we have also obtained the images for the Stokes parameters, the polarised intensity $(P)$ and position-angle $(\chi)$ images. 


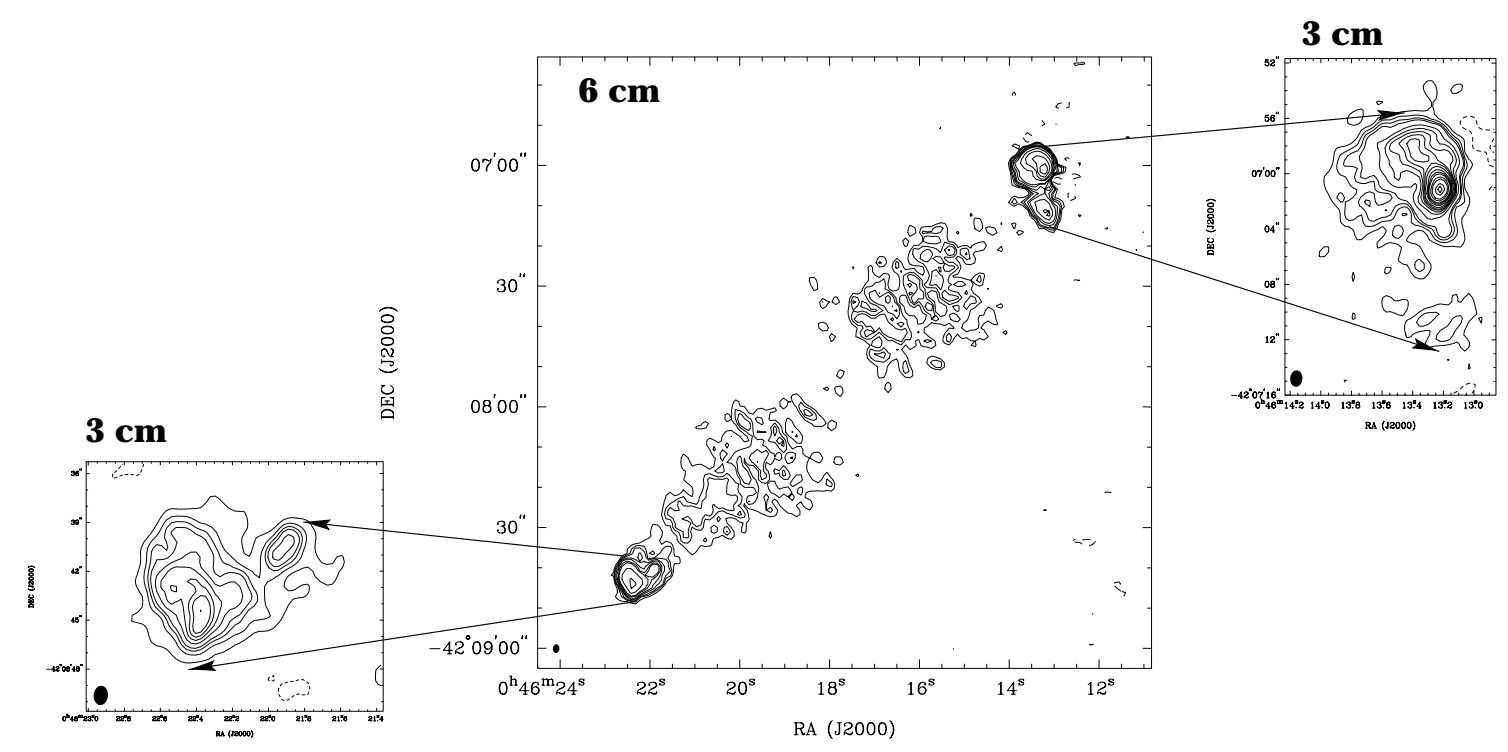

Fig. 3. Image of $0043-42$ at 6 -cm (full resolution). The contour levels are: $1.4 \times-1,1,1.5,2,3,4,6,8,16,32,64,128 \mathrm{mJy}$ beam $^{-1}$ The peak flux is 291.0 mJy beam ${ }^{-1}$. The two inserts show the images of the hot-spots at $3 \mathrm{~cm}$ (full resolution). The contour levels are: $0.6 \times-1,1,2,3,4,6,8,10,12,16,32,64,128,256$ mJy beam $^{-1}$ for the southern hot-spot and $0.8 \times-1$, $1,2,3,4,8,12,16,20,24,30,40,50,60,80,100,150,175,200,225$ mJy beam $^{-1}$ for the northern one. The peak flux is 155.0 mJy beam $^{-1}$

Table 2. ATCA observations

\begin{tabular}{|c|c|c|c|c|c|c|c|}
\hline \multirow[t]{2}{*}{ Object } & \multirow[t]{2}{*}{ Conf. } & \multirow{2}{*}{$\begin{array}{l}\lambda \\
\mathrm{cm}\end{array}$} & \multicolumn{3}{|c|}{ Resolution } & \multirow{2}{*}{$\begin{array}{c}\sigma_{\mathrm{I}} \\
\mathrm{mJy} / \text { beam }\end{array}$} & \multirow{2}{*}{$\begin{array}{c}\sigma_{\mathrm{P}} \\
\mathrm{mJy} / \text { beam }\end{array}$} \\
\hline & & & $\operatorname{arcsec}$ & $\operatorname{arcsec}$ & deg & & \\
\hline \multirow[t]{2}{*}{$0039-44$} & $6 \mathrm{~A}$ & 6 & 2.26 & 1.55 & -6.2 & 0.26 & 0.19 \\
\hline & & 3 & 1.29 & 0.91 & -6.7 & 0.18 & 0.15 \\
\hline \multirow[t]{3}{*}{$0043-42$} & $6 \mathrm{~A}+1.5 \mathrm{D}$ & 6 & 2.19 & 1.61 & -4.8 & 0.48 & $\ldots$ \\
\hline & & $6^{*}$ & 4.44 & 3.15 & -3.0 & 0.60 & 0.15 \\
\hline & & 3 & 1.19 & 0.88 & -5.1 & 0.20 & $\ldots$ \\
\hline \multirow[t]{2}{*}{$0409-75$} & $6 \mathrm{~A}$ & 6 & 2.02 & 1.25 & -14.6 & 0.81 & 0.35 \\
\hline & & 3 & 1.17 & 0.73 & -14.7 & 0.28 & 0.11 \\
\hline \multirow[t]{2}{*}{$0625-53$} & $6 \mathrm{~A}+1.5 \mathrm{D}$ & 6 & 2.03 & 1.64 & 27.3 & 0.71 & 0.16 \\
\hline & & 3 & 1.10 & 0.92 & 28.9 & 0.28 & 0.10 \\
\hline \multirow[t]{2}{*}{$1938-15$} & $6 \mathrm{~A}$ & 6 & 5.89 & 1.47 & -2.4 & 0.60 & 0.12 \\
\hline & & 3 & 3.41 & 0.88 & -1.8 & 0.40 & 0.11 \\
\hline \multirow[t]{2}{*}{$1954-55$} & $6 \mathrm{~A}$ & 6 & 2.43 & 1.29 & 78.1 & 0.53 & 0.15 \\
\hline & & 3 & 1.63 & 0.76 & 78.6 & 0.42 & 0.10 \\
\hline \multirow[t]{2}{*}{$2058-28$} & $6 \mathrm{~A}+1.5 \mathrm{D}$ & 6 & 3.17 & 1.67 & -10.7 & 0.29 & 0.15 \\
\hline & & $6^{*}$ & 7.13 & 3.56 & -9.3 & 0.47 & 0.16 \\
\hline
\end{tabular}

* Values derived from the lower resolution map (3 km maximum baseline).

The VLA observing parameters are summarised in Table 3 .

\section{Results}

Figures 1 to 14 show the final images obtained for the galaxies observed with ATCA. The electric field vectors are also shown (with the length proportional to the fractional polarization). For $1938-15$ we show the VLA image (see Fig. 9) together with the ATCA images of this source. The derived values of the fluxes and polarisation are given in Table 4 . For the $3 \mathrm{~cm}$ they are obtained from the lower resolution images. Table 4 presents for the whole source and other sub-regions (indicated in Col. 2): the fluxes at 6 and $3 \mathrm{~cm}$ (Cols. 3 and 4 respectively); the fractional polarisation at 6 and $3 \mathrm{~cm}$; the depolarization, spectral index and $R M$ between these two frequencies.

Figures 15 to 26 show the final images obtained for the galaxies observed with the VLA. The parameters derived 
Table 3. VLA observations

\begin{tabular}{|c|c|c|c|c|c|c|c|}
\hline \multirow[t]{2}{*}{ Object } & \multirow[t]{2}{*}{ Conf. } & \multirow{2}{*}{$\begin{array}{c}\lambda \\
\mathrm{cm}\end{array}$} & \multicolumn{3}{|c|}{ Resolution } & \multirow{2}{*}{$\begin{array}{c}\sigma_{\mathrm{I}} \\
\text { mJy beam }^{-1}\end{array}$} & \multirow{2}{*}{$\begin{array}{l}\sigma_{\mathrm{P}} \\
\text { mJy beam }^{-1}\end{array}$} \\
\hline & & & $\operatorname{arcsec}$ & $\operatorname{arcsec}$ & deg & & \\
\hline $0034-01$ & A & 6 & 0.41 & 0.38 & -33 & 0.04 & 0.03 \\
\hline $0035-02$ & A & 6 & 0.42 & 0.37 & -18 & 0.19 & 0.075 \\
\hline $0117-15$ & A & 6 & 0.46 & 0.31 & 1 & 0.11 & 0.06 \\
\hline $0442-28$ & A & 6 & 1.14 & 0.44 & -22 & 0.09 & 0.032 \\
\hline $0453-20$ & A & 6 & 0.89 & 0.43 & -22 & 0.30 & 0.08 \\
\hline $1602+01$ & A & 6 & 0.39 & 0.36 & -38 & 0.12 & 0.085 \\
\hline $1938-15$ & A & 6 & 0.53 & 0.29 & -20 & 0.65 & 0.21 \\
\hline $2314+03$ & A & 6 & 0.40 & 0.36 & -42 & 0.09 & 0.029 \\
\hline
\end{tabular}

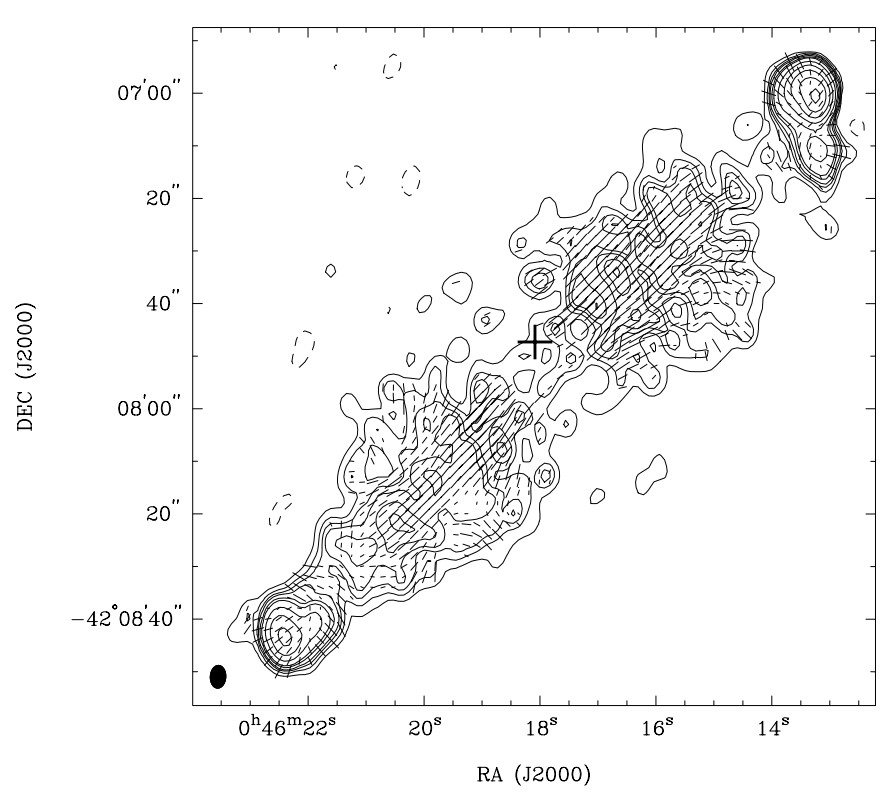

Fig. 4. Image of 0043-42 at 6-cm (low resolution) with superimposed vectors indicating the projected electric field direction. The vectors are proportional in length to the fractional polarisation ( 1 arcsec $=0.14$ ratio). The contour levels are: $1.8 \times-1,1,2,3,4,6,8,16,32,64,128,256$ mJy beam $^{-1}$. The peak flux is $545.8 \mathrm{mJy}^{\text {beam }^{-1}}$. The cross indicates the position (from di Serego et al. 1994) of the optical galaxy

from these images are listed in Table 5 . This table presents the radio fluxes and the fractional polarisation at $6 \mathrm{~cm}$ for the whole sources and their sub-regions indicated in Col. 2.

In the following section we describe in more details the results for the single objects.

\subsection{Radio galaxies observed with ATCA}

0039-44: this is a relatively small radio source with the emission dominated by the two lobes. No core or jets have been detected. Both lobes are polarised with the eastern one showing an higher fractional polarisation compared to the western side. The eastern lobe is slightly depolarised while the western side is consistent with no depolarization.

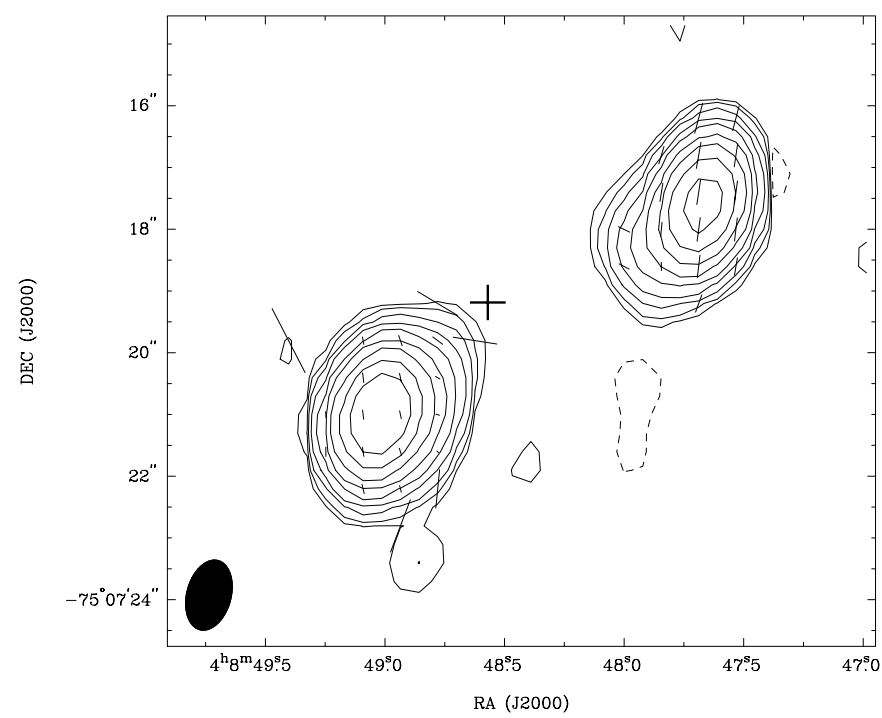

Fig. 5. Image of 0409-75 at 3-cm (full resolution) with superimposed vectors indicating the projected electric field direction. The vectors are proportional in length to the fractional polarisation $(1 \operatorname{arcsec}=0.22$ ratio $)$. The contour levels are: $2.0 \times-1$, $1,2,4,8,16,32,64,128,256$ mJy beam $^{-1}$. The peak flux is $1.255 \mathrm{Jy}^{\mathrm{beam}}{ }^{-1}$. The cross indicates the position (from di Serego et al. 1994) of the optical galaxy

In the optical, this galaxy has a high ionization emission line spectrum with a strong blue, polarized continuum. No extended emission lines have been observed in this object.

0043-42: this is a very extended source with a typical FRII morphology. It was previously mapped by Duncan \& Sproats (1992) and, at lower resolution (843 MHz with the Molonglo Synthesis Telescope, MOST), by Jones \& McAdam (1992).

Unfortunately, at 3-cm most of the extended emission is resolved out at the resolution of our data. Thus, only the hot-spots could be imaged at this frequency and they are shown in the inserts of Fig. 3 . At $6 \mathrm{~cm}$ no radio core has been detected. The polarisation is very uniform in the lobes with the electric field well aligned with the position of the radio axis. 


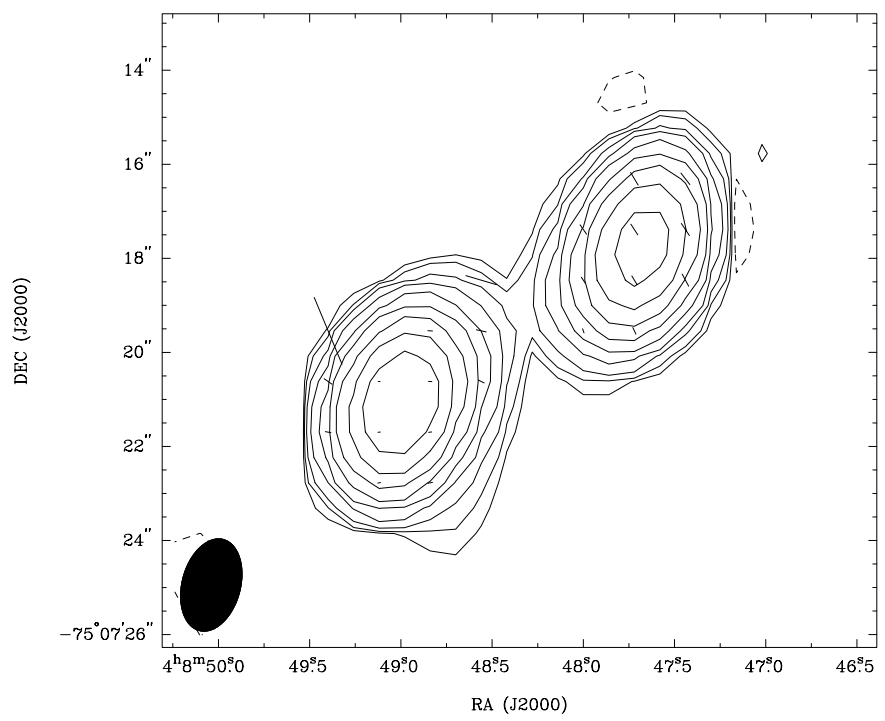

Fig. 6. Image of $0409-75$ at $6-\mathrm{cm}$ with superimposed vectors indicating the projected electric field direction. The vectors are proportional in length to the fractional polarisation ( 1 arcsec $=0.15$ ratio). The contour levels are: $4.0 \times-1,1,2,4,8,16,32$, $64,128,256 \mathrm{mJy}_{\text {beam }^{-1}}$. The peak flux is $2.349 \mathrm{Jy} \mathrm{beam}^{-1}$

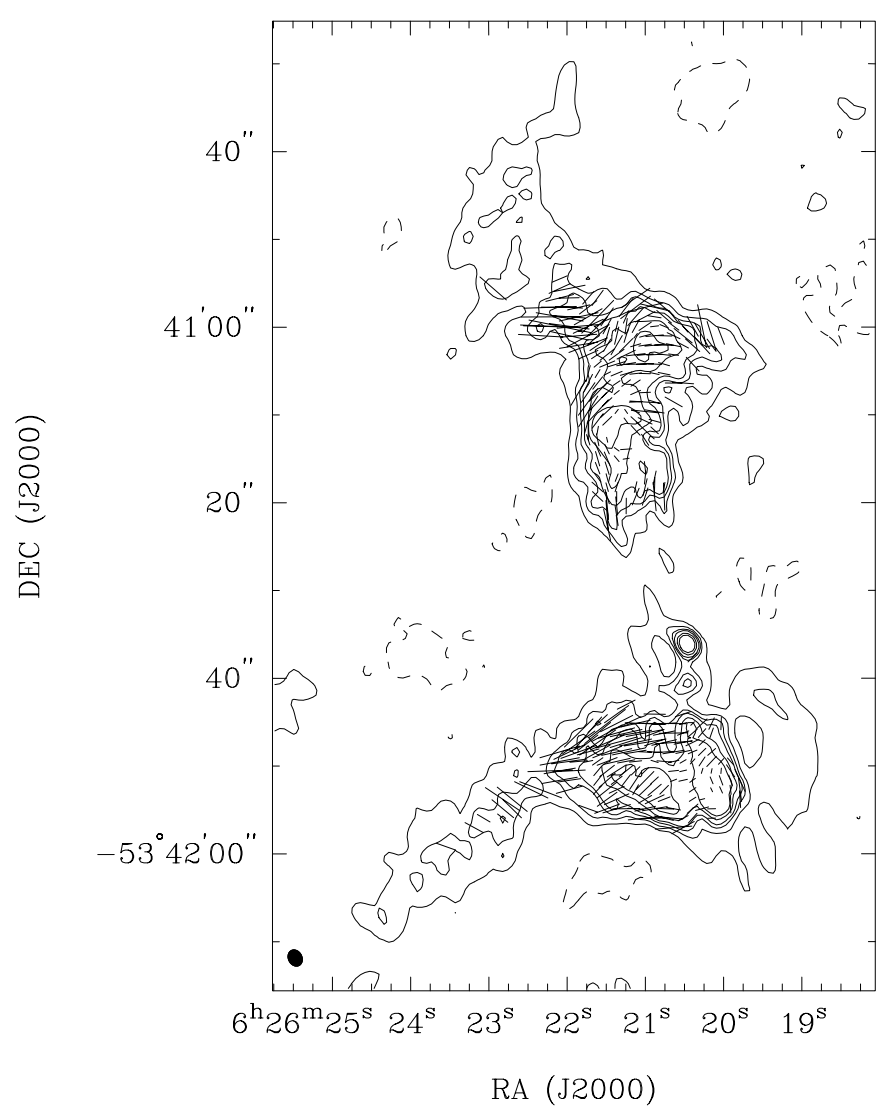

Fig. 7. Image of $0625-53$ at 6 -cm with superimposed vectors indicating the projected electric field direction. The vectors are proportional in length to the fractional polarisation ( 1 arcsec $=0.07$ ratio). The contour levels are: $1.5 \times-1,1,2,3,4,6$, $8 \mathrm{mJy}$ beam $^{-1}$. The peak flux is $22.0 \mathrm{mJy} \mathrm{beam}^{-1}$

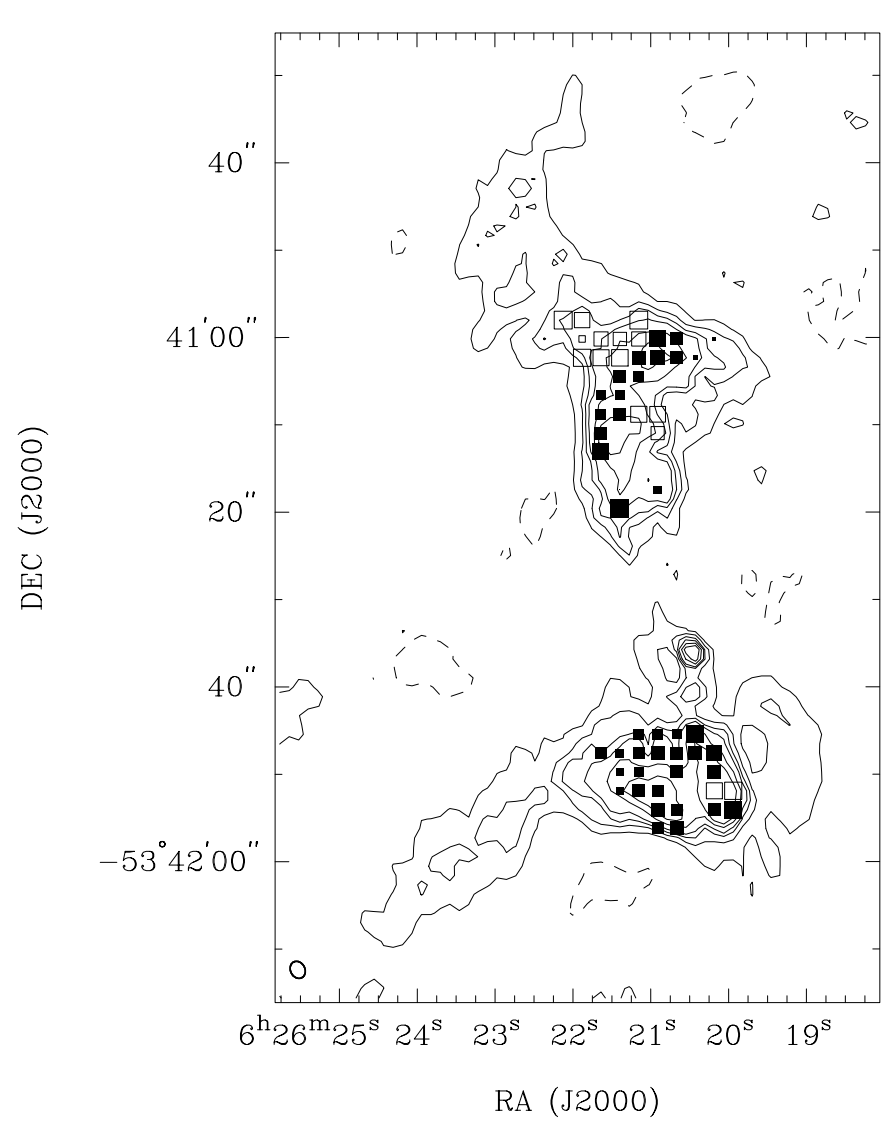

Fig. 8. Image of $0625-53$ at 6 -cm with superimposed boxes proportional to the rotation measure $(\mathrm{RM}, 1 \operatorname{arcsec}=543 \mathrm{rad}$ $\mathrm{m}^{-2}$ ). Filled squares represent positive numbers for the RM while empty squares represent negative numbers

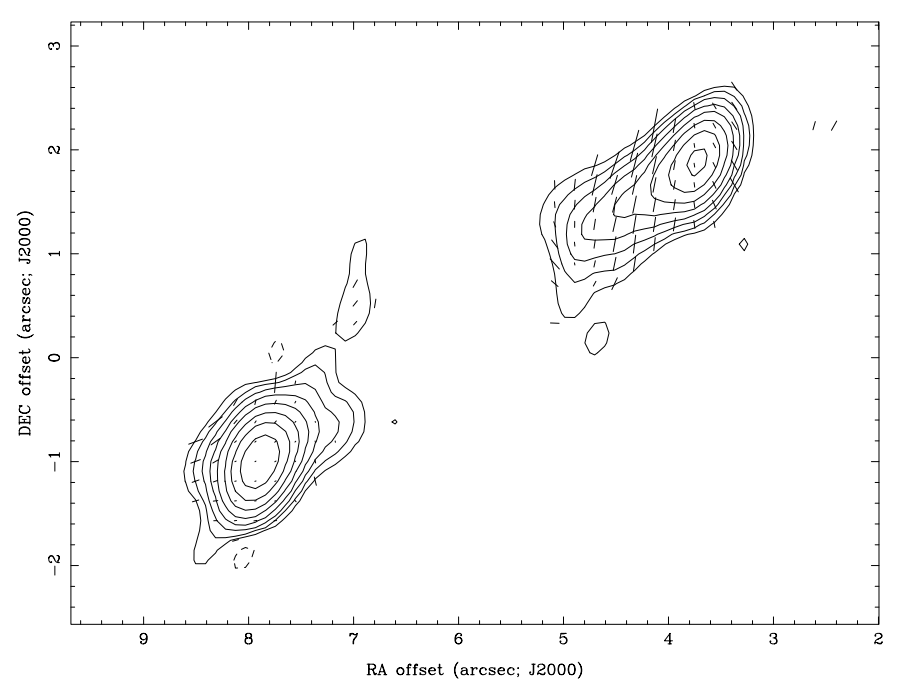

Fig. 9. Image of 1938-15 at 6-cm (VLA data) with superimposed vectors indicating the projected electric field direction. The vectors are proportional in length to the fractional polarisation $(1 \operatorname{arcsec}=1.05$ ratio $)$. The contour levels are: $5.0 \times-1$, $1,2,4,8,16,32,64,128 \mathrm{mJy}^{\text {beam }^{-1}}$. The peak flux is $1.160 \mathrm{Jy} \mathrm{beam}^{-1}$ 

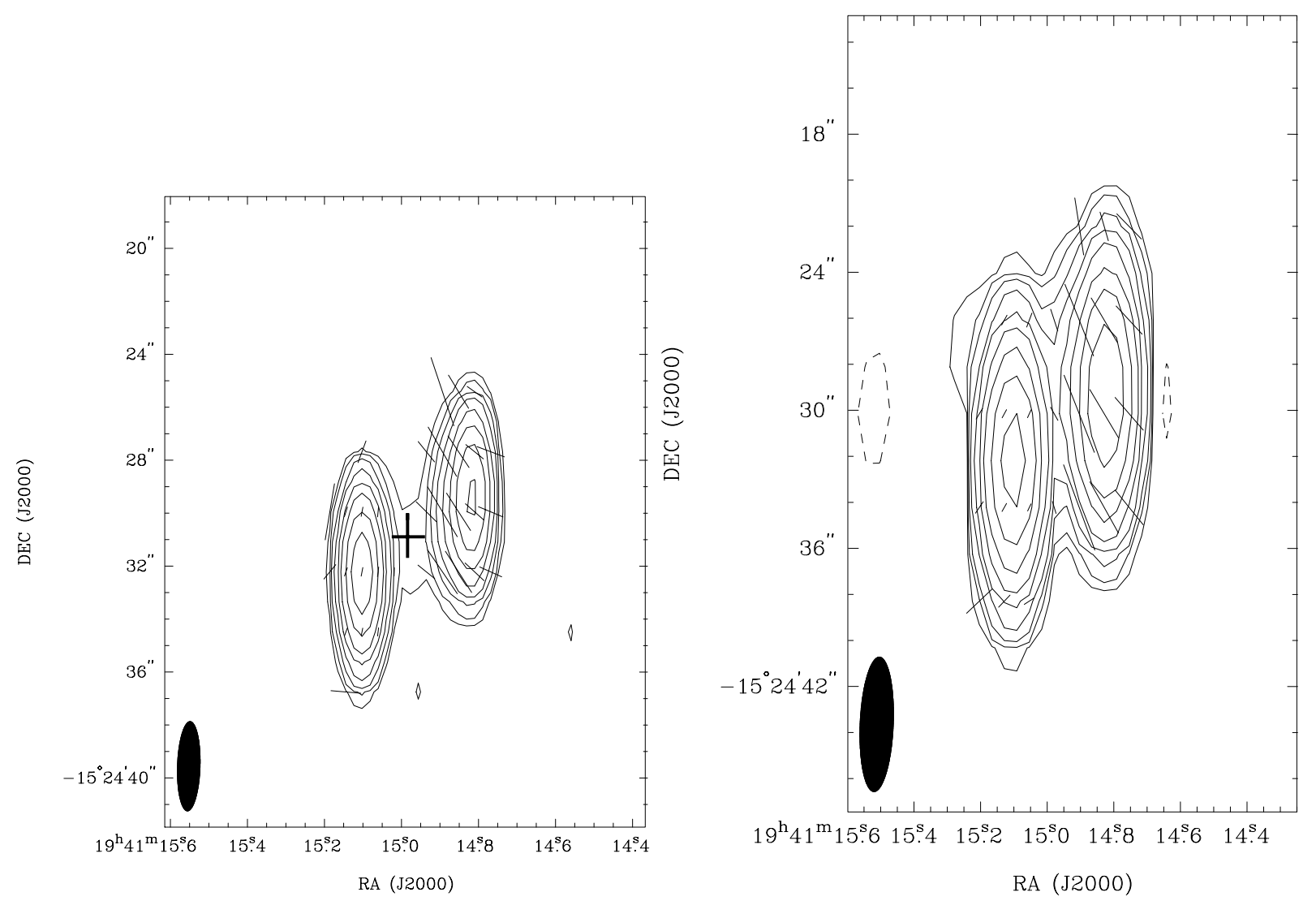

Fig. 10. (Left) ATCA image of 1938-15 at 3-cm (full resolution) with superimposed vectors indicating the projected electric field direction. The vectors are proportional in length to the fractional polarisation $(1 \operatorname{arcsec}=0.07$ ratio $)$. The contour levels are: $3.0 \times-1,1,2,4,8,16,32,64,128 \mathrm{mJy}_{\text {beam }}{ }^{-1}$. The peak flux is 658.5 mJy beam $^{-1}$; (Right) ATCA image of $1938-15$ at 6 -cm with superimposed vectors indicating the projected electric field direction. The vectors are proportional in length to the fractional polarisation. The contour levels are: $3.5 \times-1,1,2,4,8,16,32,64,128,256 \mathrm{mJy} \mathrm{beam}^{-1}$. The peak flux is $1.240 \mathrm{Jy}$ beam $^{-1}$. The cross indicates the position (from Prestage \& Peacock 1983) of the optical galaxy

In the optical it shows only weak, low-ionization emission lines and its continuum appears to be typical of early-type galaxies. Thus, this is an example of FRII (and powerful) radio galaxies with significantly weaker emission lines that expected from the radio power-emission line luminosity correlation.

0409-75: this FRII is the highest red-shift object in the sample and one of the most powerful source in the southern hemisphere (Alvarez et al. 1993). It was previously mapped by Duncan \& Sproats (1992).

It is quite a small radio source with the radio emission dominated by two bright lobes. Both lobes have an high depolarization, slightly higher in the eastern lobe. Also the rotation measure is large especially in the eastern lobe.

Interestingly, the ionization state is low for such a powerful radio galaxy: [O III] $\lambda 5007$ is barely detected while $[\mathrm{OII}] \lambda 3727$ is strong. However the continuum is bluer than an elliptical galaxy at the same red-shift (Dickson 1997). According to Tadhunter et al. (1993) the
[O II] $\lambda 3727$ line is extended but this does not seem to be the case in [O III] $\lambda 5007$.

0625-53: This is a FRI galaxy associated with a dumbbell galaxy (Lilly \& Prestage 1987; Gregorini et al. 1994) that is also the brightest member of the cluster Abell 3391. At the resolution of our observations, it shows a wideangle tail (WAT) structure with two tails sharply bending at about 20 arcsec north and 40 arcsec south of the nucleus. This morphology is also confirmed by the $13-\mathrm{cm}$ ATCA image presented by Otani et al. (1998). From an $\mathrm{X}$-ray/radio comparison, these authors found evidence for a possibly strong interaction between the radio jets and the surrounding material. The jets are deflected possibly as the result of pressure gradients or winds in the intracluster medium and they seem to "escape" into regions of lower X-ray brightness. This galaxy has also been observed at lower resolution by Gregorini et al. (1994). In their image, the northern tail is even more prominent and a low-brightness diffuse region is also observed as a western extension (for $\sim 45 \operatorname{arcsec}$ ) to the southern lobe. 
The two lobes show a similar fractional polarisation at both 6 and $3 \mathrm{~cm}$ and they both show no significant depolarisation. The $R M$ is quite uniform in the southern lobe but a large range of $R M$ values is observed in the northern lobe (see Fig. 8) with the central region around $R M \sim 350 \mathrm{rad} \mathrm{m}^{-2}$, while the northern tail is showing values of $\sim-430 \mathrm{rad} \mathrm{m}^{-2}$. There is the possibility that the values are continuous at the step and the reason for the "apparent" jump is the $n \pi$ ambiguity in the position angle: only a study of the polarisation at other frequencies will be able to confirm this jump in $R M$. However, it may be worth noticing that, by looking at Fig. 7 in Otani et al. (1998) (i.e. the radio/X-ray overlay), the jump in $R M$ occurs in the region where the radio emission seems to "escape" the brighter X-ray emission.

1938-15: this is another small source dominated by the radio emission from two lobes. No core or jets have been detected. It shows quite a strong asymmetry in the depolarization: the eastern lobe has the lower fractional polarisation and the stronger depolarization.

This galaxy has a high ionization emission line spectrum. From the new optical spectra (Dickson 1997) this galaxy is now classified as a Broad Line Radio Galaxy (BLRG) because it shows a prominent broad component in the $\operatorname{MgII} \lambda 2800 \AA$ and $\mathrm{H} \beta$. As with many other BLRG, it is detected in X-ray by ROSAT (Siebert et al. 1996).

1954-55: this is an FRI source comprising two jet-like structures. When observed at low frequency and low resolution (Jones \& Mc Adams 1992), this object is embedded in an elongated and bent low-brightness halo extended more than 5 arcmin. The integrated spectral index we find is much steeper than from the single-dish observations (see Table 1) and this is probably due to resolved emission in the $3 \mathrm{~cm}$ image.

Neither jet shows evidence for significant depolarization. The rotation measure is quite uniform in the southern lobe $\left(\sim 126 \mathrm{rad} \mathrm{m}^{-2}\right)$. In the northern lobe, most of the lobe shows a value of around $18 \mathrm{rad} \mathrm{m}^{-2}$ except in a region (south-west) where the values are around $\sim-83$ $\mathrm{rad} \mathrm{m}^{-2}$.

2058-28: the morphology of this FRI source is better defined than in the previously available radio image. A strong jet is also now observed. The difference with the previous radio image is likely due to the short integration of the previous observations. Unfortunately, this galaxy could not be imaged properly at $3-\mathrm{cm}$ and therefore we do not show the $3 \mathrm{~cm}$ data here. A low resolution radio image was obtained by Christiansen et al. (1977) and shows that the source is embedded in a low brightness halo, extended more than 8 arcmin, which is not visible in our image.

Two diffuse lobes without hot-spots are observed. The polarisation in these lobes has a ring-like shape and the position angle of the electric field is radial in both the lobes.

2058-28 has a core flux density higher than in the previous VLA $6 \mathrm{~cm}$ data. The detected difference is likely to

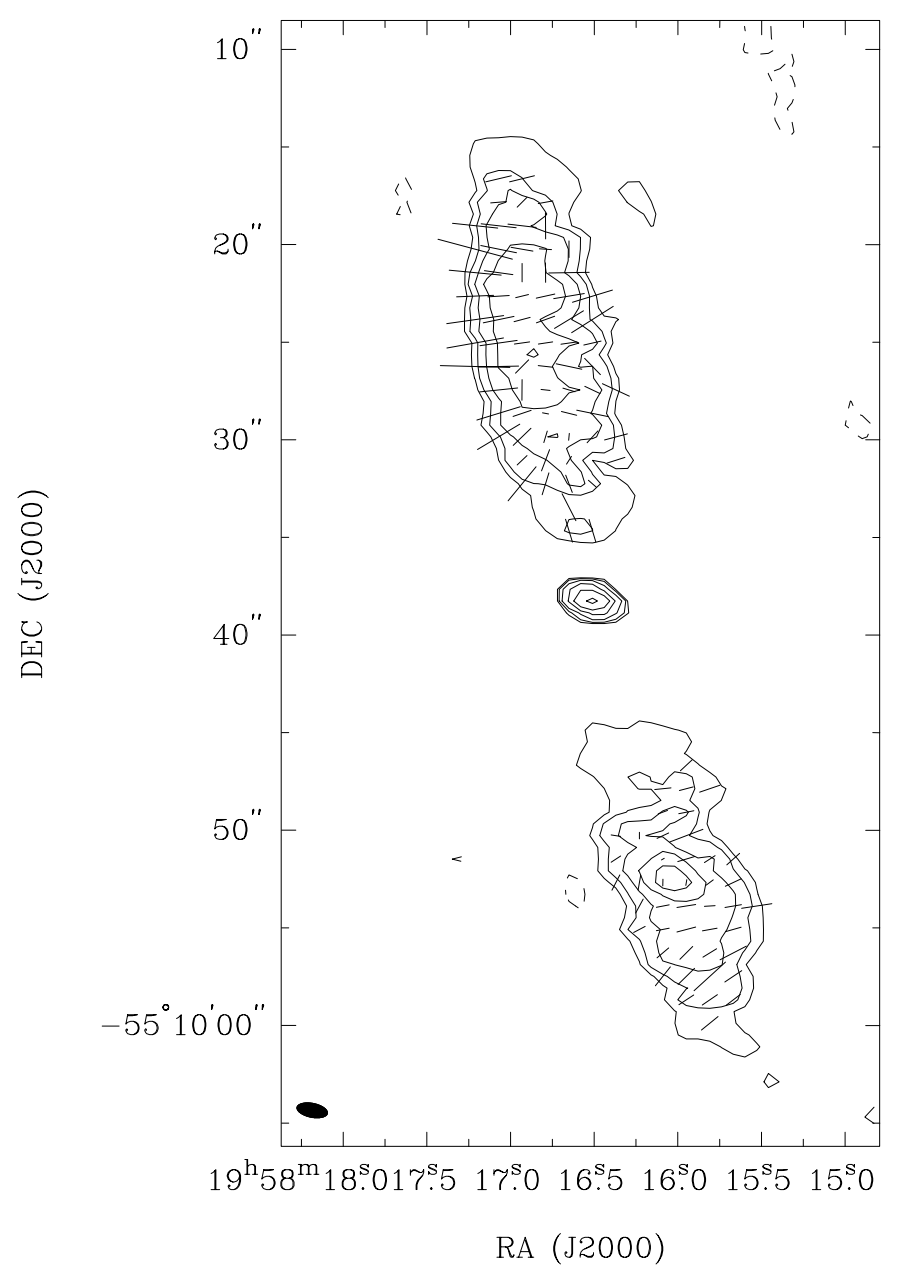

Fig. 11. Image of 1954-55 at 3-cm (full resolution) with superimposed vectors indicating the projected electric field direction. The vectors are proportional in length to the fractional polarisation ( $1 \operatorname{arcsec}=0.54$ ratio). The contour levels are: $1.3 \times-1,1,2,4,8,16,32 \mathrm{mJy}^{\text {beam }^{-1}}$. The peak flux is $54.5 \mathrm{mJy}^{\text {beam }^{-1}}$

be due to the old value of the radio core being unreliable, for the reasons mentioned above, more than variability in the core flux. Furthermore, the new core flux density is consistent with the PTI data derived at $13 \mathrm{~cm}$ (Morganti et al. 1997a).

In the optical it shows only weak, low-ionization emission lines and its continuum appears to be typical of earlytype galaxies.

\subsection{Radio galaxies observed with VLA}

0034-01 (3C 15): this source is now resolved into a prominent one-sided jet, while only some structure is detected in the two lobes (due to the relatively high resolution). The southern lobe is the brighter one but only shows a "warmer" spot (as perhaps expected looking at the low resolution image in Morganti et al. 1993). This galaxy has been observed at $3.6 \mathrm{~cm}$ by Leahy et al. (1997) who define 
Table 4.

\begin{tabular}{|c|c|c|c|c|c|c|c|c|}
\hline Object & & $\begin{array}{c}S_{6 \mathrm{~cm}} \\
\mathrm{Jy}\end{array}$ & $\begin{array}{c}S_{3 \mathrm{~cm}} \\
\mathrm{Jy}\end{array}$ & $\begin{array}{c}m_{6 \mathrm{~cm}} \\
\%\end{array}$ & $\begin{array}{c}m_{3 \mathrm{~cm}} \\
\%\end{array}$ & $\mathrm{DP}_{3 \mathrm{~cm}}^{6 \mathrm{~cm}}$ & $\alpha_{3 \mathrm{~cm}}^{6 \mathrm{~cm}}$ & $\begin{array}{c}\mathrm{RM} \\
\operatorname{rad} \mathrm{m}^{-2}\end{array}$ \\
\hline \multirow[t]{3}{*}{$0039-44$} & Total & 1.169 & 0.606 & 7.5 & 6.7 & $\ldots$ & 1.20 & $\ldots$ \\
\hline & E lobe & 0.678 & 0.344 & 8.0 & 10.2 & 0.79 & 1.24 & -27 \\
\hline & W lobe & 0.486 & 0.261 & 3.7 & 2.7 & 1.35 & 1.14 & -32 \\
\hline \multirow[t]{5}{*}{$0043-42^{*}$} & Total & 2.776 & $\ldots$ & 30.3 & $\ldots$ & $\ldots$ & $\ldots$ & $\ldots$ \\
\hline & HS North & 1.182 & $\ldots$ & 20.1 & $\ldots$ & $\ldots$ & $\ldots$ & $\ldots$ \\
\hline & Ext. North & 0.507 & $\ldots$ & 37.1 & $\ldots$ & $\ldots$ & $\ldots$ & $\ldots$ \\
\hline & HS South & 0.397 & $\ldots$ & 20.0 & $\ldots$ & $\ldots$ & $\ldots$ & $\ldots$ \\
\hline & Ext. South & 0.550 & $\ldots$ & 28.9 & $\ldots$ & $\ldots$ & $\ldots$ & $\ldots$ \\
\hline \multirow[t]{3}{*}{$0409-75$} & Total & 4.508 & 2.315 & 2.5 & 6.6 & 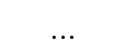 & 1.22 & $\ldots$ \\
\hline & E lobe & 2.671 & 1.459 & 1.1 & 3.4 & 0.30 & 1.10 & +522 \\
\hline & W lobe & 1.843 & 0.895 & 4.5 & 9.3 & 0.46 & 1.32 & +235 \\
\hline \multirow[t]{4}{*}{$0625-53$} & Totale & 1.543 & 0.787 & 15.7 & 15.1 & 0.96 & 1.23 & +150 \\
\hline & Core & 0.025 & 0.019 & $\ldots$ & $\ldots$ & $\ldots$ & 0.47 & $\ldots$ \\
\hline & N Lobe & 0.747 & 0.383 & 12.1 & 14.8 & 0.91 & 1.22 & $+43^{a}$ \\
\hline & S Lobe & 0.691 & 0.351 & 12.6 & 12.3 & 1.02 & 1.24 & +293 \\
\hline \multirow[t]{3}{*}{$1938-15$} & Total & 2.377 & 1.263 & 6.3 & 8.8 & $\ldots$ & 1.16 & $\ldots$ \\
\hline & E lobe & 1.370 & 0.721 & 2.1 & 3.8 & 0.53 & 1.17 & -105 \\
\hline & W lobe & 1.032 & 0.549 & 11.6 & 13.7 & 0.98 & 1.15 & -79 \\
\hline \multirow[t]{4}{*}{$1954-55$} & Total & 1.630 & 0.559 & 32.7 & 34.0 & $\ldots$ & 1.95 & +33 \\
\hline & Core & 0.055 & 0.052 & $\ldots$ & $\ldots$ & $\ldots$ & 0.10 & $\ldots$ \\
\hline & $\mathrm{N}$ lobe & 1.340 & 0.351 & 39.1 & 39.8 & 0.98 & 2.45 & $0^{a}$ \\
\hline & S lobe & 0.608 & 0.165 & 26.5 & 23.2 & 1.06 & 2.38 & 126 \\
\hline \multirow[t]{5}{*}{$2058-28^{*}$} & Total & 1.216 & $\ldots$ & 28.0 & $\ldots$ & $\ldots$ & $\ldots$ & $\ldots$ \\
\hline & Core & 0.123 & $\ldots$ & $\ldots$ & $\ldots$ & $\ldots$ & $\ldots$ & $\ldots$ \\
\hline & S lobe & 0.591 & $\ldots$ & 25.3 & $\ldots$ & $\ldots$ & $\ldots$ & $\ldots$ \\
\hline & $\mathrm{S}$ jet & 0.069 & $\ldots$ & 13.4 & $\ldots$ & $\ldots$ & $\ldots$ & $\ldots$ \\
\hline & $\mathrm{N}$ lobe & 0.440 & $\ldots$ & 33.4 & $\ldots$ & $\ldots$ & $\ldots$ & $\ldots$ \\
\hline
\end{tabular}

* Values derived from the lower resolution map (3 $\mathrm{km}$ maximum baseline).

${ }^{a}$ See notes to the sources (Sect. 3.1).

its structure as intermediate between FR classes I and II of FR, although its radio luminosity is typical of class II galaxies (indeed it was classified as FRII in Morganti et al. 1993). Knots are detected along the jet as in the $3.6 \mathrm{~cm}$ images.

The core flux density measured in the new image is much lower than from the $6 \mathrm{~cm}$ VLA data: this is probably due to the fact that low resolution of the previous observations (about 3 arcsec) included the base of the strong jet in what was defined as the core.

The electric field in the jet is perpendicular to the jet axis in the first blob (closer to the nucleus) and then become parallel to the jet, although a different position angle seems to be characteristics of the regions at the edge. This is likely due to effect of shear layer as often found in jets in FRI and described in detailed by Laing et al. (1996).
In the optical only a weak [O III] $\lambda 5007$ emission has been detected from this source (Tadhunter et al. 1993), and the continuum is typical of early-type galaxies.

0035-02 (3C 17): this radio galaxy presented a very peculiar radio morphology in our previous low resolution radio image (Morganti et al. 1993). The new high resolution image clarifys the real structure of this source. On the south-east side of the nucleus a very bent jet is observed. The first part of the jet is dominated by a bright blob. On the western side a lobe structure is observed with a ring-like shape, i.e. because of the minimum in intensity in the centre. Also this could be a very bent jet seen in a particular position angle. A VLBA map (Venturi et al. 1996) shows as on the milliarcsec scale a one-sided jet is observed in the eastern side and in the same position angle as the bright blob observed in our VLA map. 


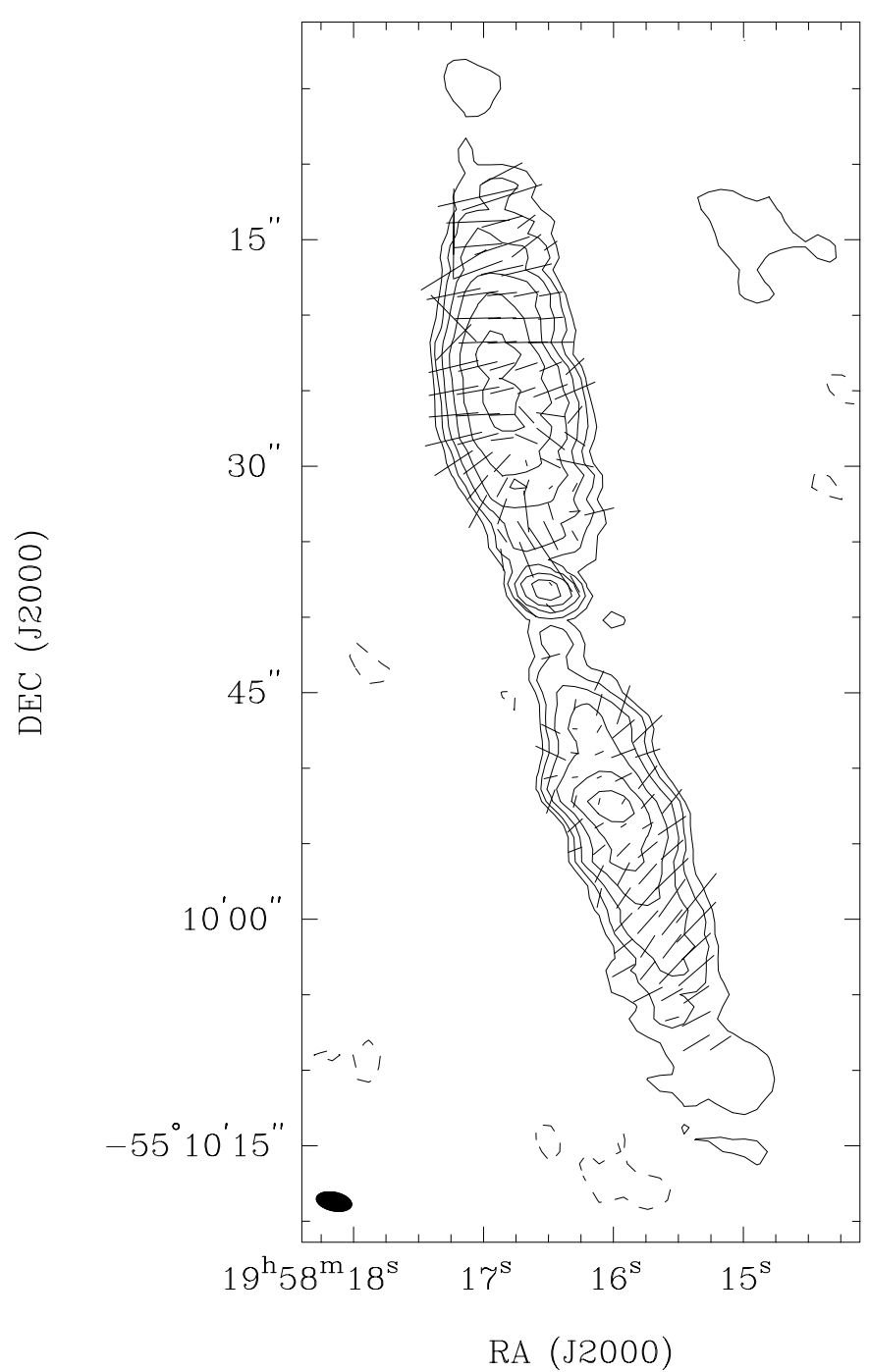

Fig. 12. Image of $1954-55$ at 6 -cm with superimposed vectors indicating the projected electric field direction. The vectors are proportional in length to the fractional polarisation ( 1 arcsec $=0.029$ ratio). The contour levels are: $1.6 \times-1,1,2,4,8,16$, $32 \mathrm{mJy}_{\text {beam }^{-1}}$. The peak flux is $94.0 \mathrm{mJy}^{\text {beam }}{ }^{-1}$

In this galaxy, the $\mathrm{H} \alpha$ emission line has a strong broad component (Dickson 1997) and the [O II] $\lambda 3727$ and [O III] $\lambda 5007$ emission lines are extended. There is also evidence for significant optical polarization in the nucleus of the galaxy (Tadhunter et al. 1997). This object has been detected in X-rays (Siebert et al. 1996), as have most of the BLRG in our sample.

Given the optical polarization properties (Tadhunter et al. 1997) this object may be a BL Lac-type in which the jet is pointing close to the line of sight (thus accentuating any wiggles in the jet). It also looks very similar to the southern BLRG/quasar PKS 2300-18 which has been interpreted in terms of precession.

0117-15 (3C 38): is a double lobed radio galaxy with a typical FRII structure. No core has been detected. Both lobes have clear hot spots and the southern lobe shows a

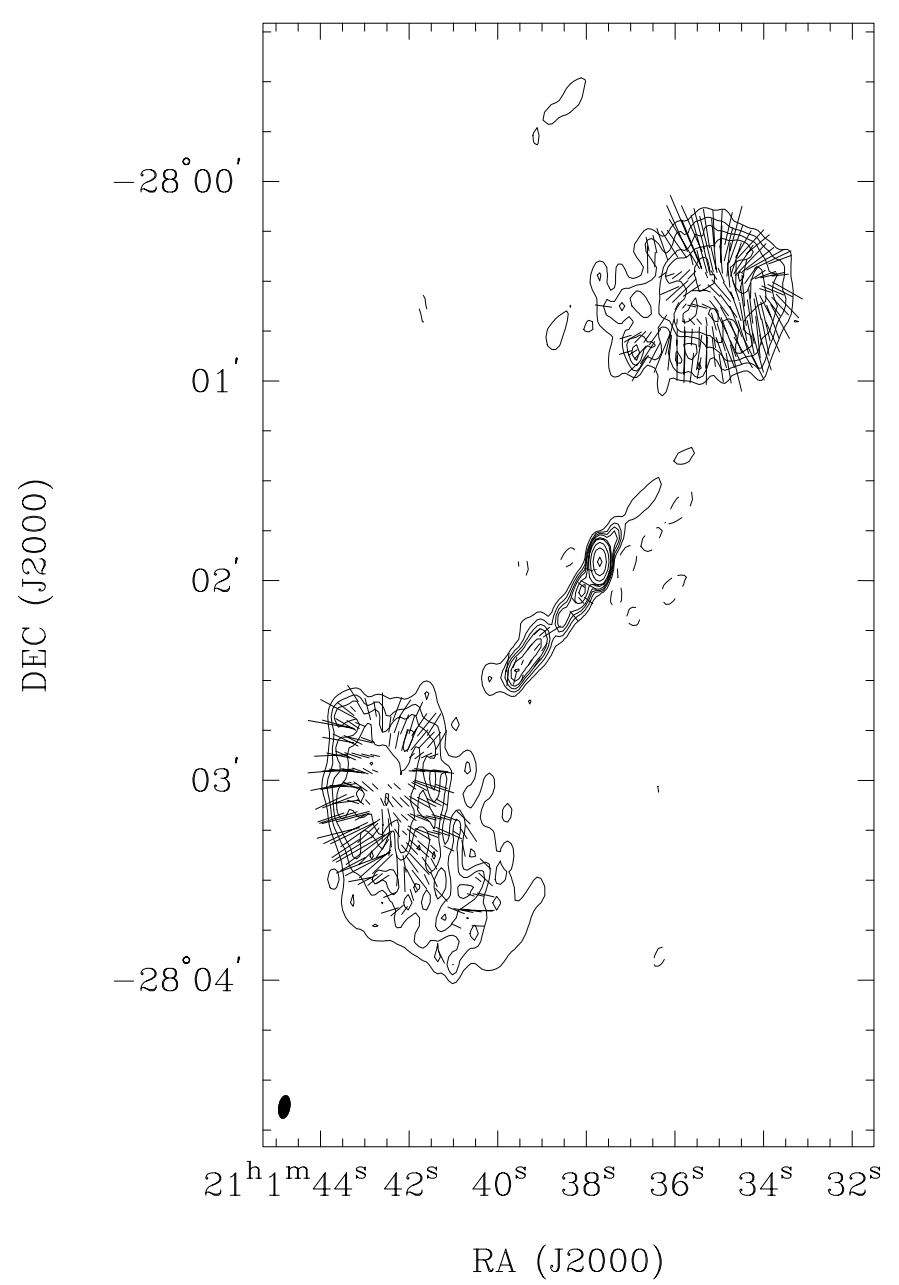

Fig. 13. Image of 2058-28 at 6-cm (low resolution) with superimposed vectors indicating the projected electric field direction. The vectors are proportional in length to the fractional polarisation ( $1 \operatorname{arcsec}=0.04$ ratio). The contour levels are: $1.5 \times-1,1,2,3,4,6,8,16,32,64$ mJy beam $^{-1}$. The peak flux is $112.0 \mathrm{mJy}^{\text {beam }}{ }^{-1}$

clear bend in a direction almost perpendicular to the line connecting the two lobes. Similar structure detected, e.g., in 3C 326 (Ekers et al. 1978) has been explained as result of a precessing beam. An alternative explanation has been suggested from a systematic study of bridges in double radio sources by Leahy \& Williams (1984). The sharp distortion often observed in these sources can be expected as consequence of the interaction between the strong back-flow and the galactic atmosphere if the jet is much lighter than the confining medium.

In the optical, this galaxy has a high ionization spectrum with both the continuum and the line emission extended in the north-east direction. The continuum shows a strong UV/blue excess (Dickson 1997) and substantial optical polarisation (Tadhunter et al. 1997). The radio lobe on the same side as the extended emission lines is closer to the nucleus and has the lower fractional polarisation. 


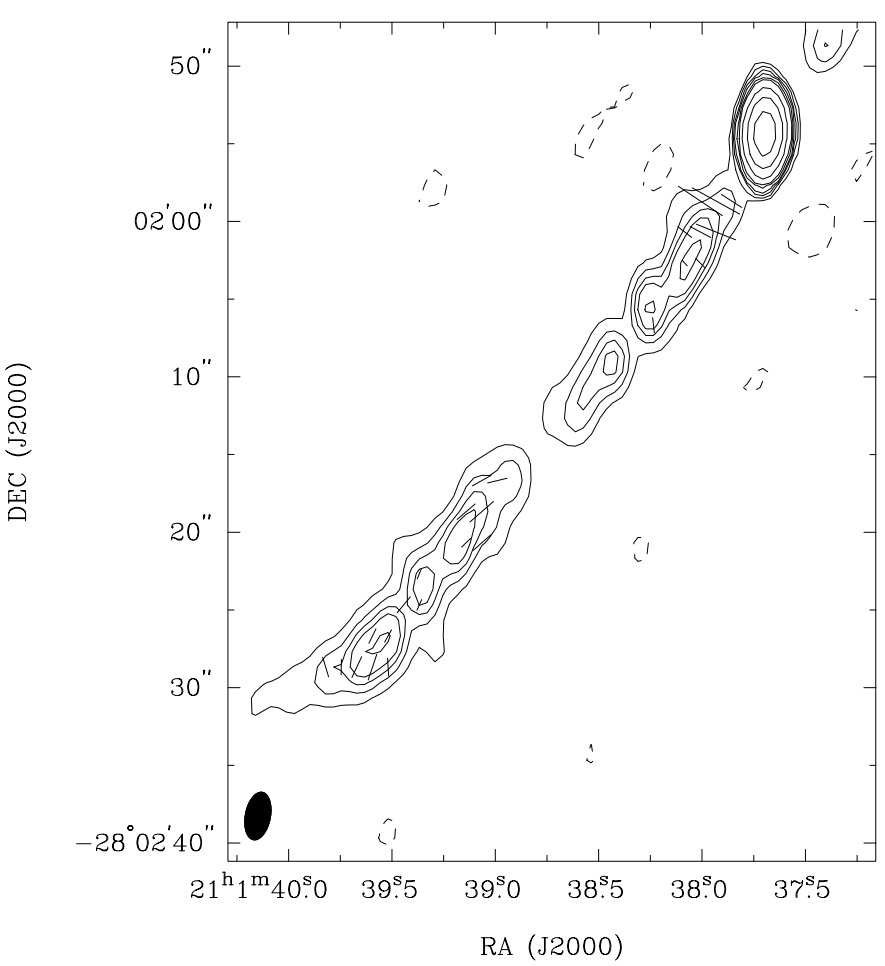

Fig. 14. Zoom-in of the jet in 2058-28 at 6-cm (full resolution) with superimposed vectors indicating the projected electric field direction. The vectors are proportional in length to the fractional polarisation $(1 \operatorname{arcsec}=0.13$ ratio $)$. The contour levels are: $1.0 \times-1,1,2,3,4,6,8,16,32,64 \mathrm{mJy}_{\text {beam }}{ }^{-1}$. The peak flux is 100.6 mJy beam $^{-1}$

This may indicate a higher depolarization but confirmation will require observations at different frequencies.

0442-28: the new radio observations for this FRII radio galaxy shows a complex morphology for its radio lobes. A core has been detected but no radio jets.

This object has strong, narrow emission lines (Tadhunter et al. 1993). It is also detected in X-rays (Siebert et al. 1996), where it appears to be extended, even though this source is not known to be associated with a cluster.

0453-20: from the new radio image this source appears as double lobed with a clear jet in the north-west direction ending with an hot spot. In the southern part no jet has been detected and a "warm-spot" is visible at the edge of the lobe. The electric field is parallel to the direction of the jet and becomes radial at the edge of the lobe.

From Tadhunter et al. (1993) no optical lines are detected in this galaxy and the continuum appears typical of early-type galaxies.

$1602+01$ (3C 327.1): this source is dominated by a one sided knotty jet on the south-east side. This galaxy was

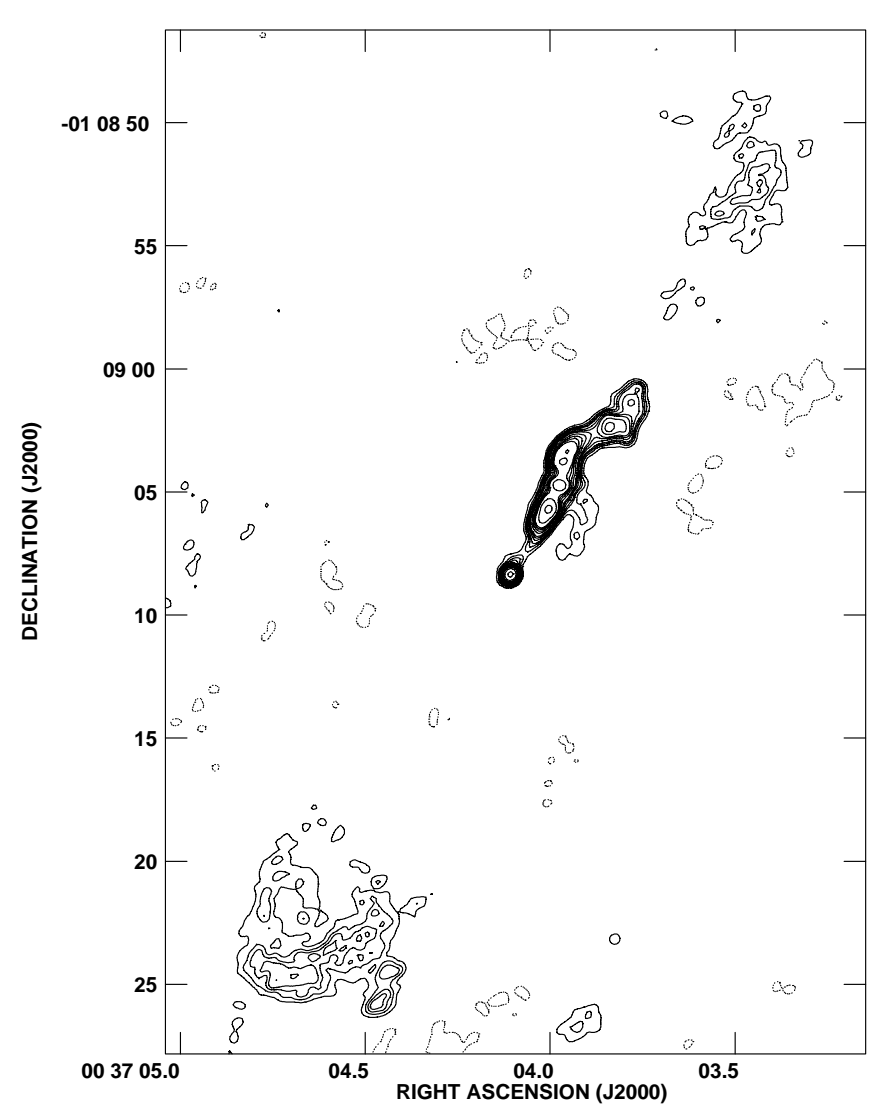

Fig. 15. Image of $0034-01$ at 6 -cm (VLA data). The contour levels are: $0.25 \times-1,1,2,3,4,6,8,10,12,14,16,20,25$, $30,40,50,75,100$ mJy beam $^{-1}$. The peak flux is $31.1 \mathrm{mJy}$ beam $^{-1}$

also observed by Baum et al. (1988) with the B array at $6 \mathrm{~cm}$, and by Hes (1995) at $8.4 \mathrm{GHz}$.

In the radio, $1602+01$ shows clear morphological similarities with $0035-02$. Similarities are also present in the optical spectrum of these objects. In fact, in the optical 1602+01 has a high ionization emission line spectrum showing (as 0035-02) a broad component of the $\mathrm{H} \beta$ emission (Dickson 1997). The [O II] $\lambda 3727$ and [O III] $\lambda 5007$ may be slightly extended. As with most of the BLRG, this galaxy has been detected in X-ray emission.

1938-15: this object has been also observed with the ATCA and described above. From the higher resolution of the VLA map (Fig. 9) it is possible to see that the western lobe is actually extended while the eastern one is only slightly resolved. Also at the higher resolution the east lobe shows a low polarisation (as in the lower resolution ATCA image). The west lobe (now better resolved) shows a higher fractional polarisation in the VLA image ( $\sim 29 \%$ ), indicating possible depolarization due to the beam at the resolution of ATCA.

2314+03 (3C 459): this is another small source of this sample also studied before by Ulvestad (1985). It is dominated by a strong core and two lobes. The eastern lobe is quite compact and much closer to the nucleus compared 
Table 5.

\begin{tabular}{|c|c|c|c|}
\hline Object & & $\begin{array}{c}\mathrm{S}_{6 \mathrm{~cm}} \\
\mathrm{Jy}\end{array}$ & $\begin{array}{c}m_{6 \mathrm{~cm}} \\
\%\end{array}$ \\
\hline \multirow[t]{4}{*}{$0034-01$} & core & 0.032 & $\ldots$ \\
\hline & jet & 0.292 & 25.0 \\
\hline & $\mathrm{N}$ lobe & 0.049 & \\
\hline & S lobe & 0.109 & 53.2 \\
\hline \multirow[t]{4}{*}{ 0035-02 } & Total & 1.279 & 33.4 \\
\hline & core & 0.530 & \\
\hline & E jet & 0.475 & 30.0 \\
\hline & W lobe & 0.275 & 40.3 \\
\hline \multirow[t]{3}{*}{$0117-15$} & Total & 1.336 & 25.2 \\
\hline & N-E lobe & 0.985 & 15.4 \\
\hline & S-W lobe & 0.347 & 35.2 \\
\hline \multirow[t]{3}{*}{$0442-28$} & core & 0.039 & . \\
\hline & $\mathrm{N}$ lobe & 0.573 & 25.6 \\
\hline & $\mathrm{S}$ lobe & 0.386 & 37.8 \\
\hline \multirow[t]{4}{*}{$0453-20$} & Total & 0.952 & 47.4 \\
\hline & core & 0.034 & $\ldots$ \\
\hline & $\mathrm{N}$ lobe & 0.546 & 47.1 \\
\hline & S lobe & 0.323 & 48.4 \\
\hline \multirow[t]{4}{*}{$1602+01$} & Total & 0.945 & $\ldots$ \\
\hline & core & 0.080 & $\ldots$ \\
\hline & E lobe & 0.423 & 21.6 \\
\hline & W lobe & 0.441 & 16.7 \\
\hline \multirow[t]{4}{*}{$1938-15$} & Total & 2.947 & 11.4 \\
\hline & E lobe & 1.729 & 2.8 \\
\hline & W hot-spot & 1.094 & 9.2 \\
\hline & W lobe & 1.269 & 28.5 \\
\hline \multirow[t]{4}{*}{$2314+03$} & Total & 1.294 & 14.4 \\
\hline & core & 0.425 & 2.0 \\
\hline & E lobe & 0.608 & 2.0 \\
\hline & W lobe & 0.260 & 19.2 \\
\hline
\end{tabular}

to the western one. A strong asymmetry in the polarisation can be seen between the two lobes with the eastern one much less polarised (only $2 \%$ ) than the western one consistent with what was found by Ulvestad (1985). Also in this object, as in 0034-01, the new core flux density is lower than in the previous VLA data (Morganti et al. 1993). Again, the difference is likely due to the difference in resolution.

In the optical this galaxy has a moderate ionization emission line spectrum and the continuum is dominated by young stars.

\section{Discussion and conclusions}

We have presented in this paper new, higher resolution radio images of a group of 14 galaxies belonging to the 2-Jy sample of radio sources. The new images improve on the data already available for these objects and, in general, the database that we are building up on the sample.

Although the radio data presented here are mainly useful in the context of the work we are doing on the sample, it is still interesting to highlight some general results that can be derived from the new data. These will be briefly described in the following sections.

\subsection{The radio cores}

From our previous studies we have found that the core dominance [i.e. ratio between the core and the extended radio fluxes $R=S_{\text {core }} /\left(S_{\text {tot }}-S_{\text {core }}\right)$, in tests of unified schemes commonly used as an indicator for the orientation of a source] appears to depend on both the morphological classification (Morganti et al. 1995) and the optical characteristics of the radio galaxies. Among the FRII radio galaxies, most Narrow Line Radio Galaxies (NLRGs) show low values of $\log R$, while BLRGs tend to have large $\log R$ (Laing et al. 1994; Morganti et al. 1997a; Hardcastle et al. 1998) supporting the idea that BLRGs are more beamed toward us.

What do the new radio data tell us? In the observed sample we have three BLRG: 0035-02, 1602+01, 1938-15 (two of them only recently classified as BLRG). Both 0035-02 and 1602+01 have very prominent cores and the core flux densities measured in the new observations are consistent with what was found before. In particular we find $R=0.33$ and $R=0.061$ for $0035-02$ and $1602+01$ respectively (if using the total fluxes taken from single dish observations, Peacock \& Wall 1985). On the other hand, in 1938-15 we do not detect any core in our higher resolution map. This sets an upper limit to the value of $R$ (core flux $\lesssim 1.5 \mathrm{mJy} ; R \sim 0.0004$ ), well below the average value for BLRG $(\sim 0.027)$. Thus, this object appears to be an exception like $0347+05\left(R_{2.3 \mathrm{GHz}}<0.0006\right)$ as pointed out by Tadhunter et al. (1998). These objects deserve some follow-up work to understand their real nature and why they show such differences from the other BLRG.

\subsection{Asymmetry in depolarization}

For the objects for which we have two frequencies available, i.e. the objects observed with ATCA, we could investigate the presence of asymmetries in the depolarization. This asymmetry has been found in 0039-44, 0409-75 and 1938-15 and it is also notable that these are among the highest redshift sources in the sample and they are all 

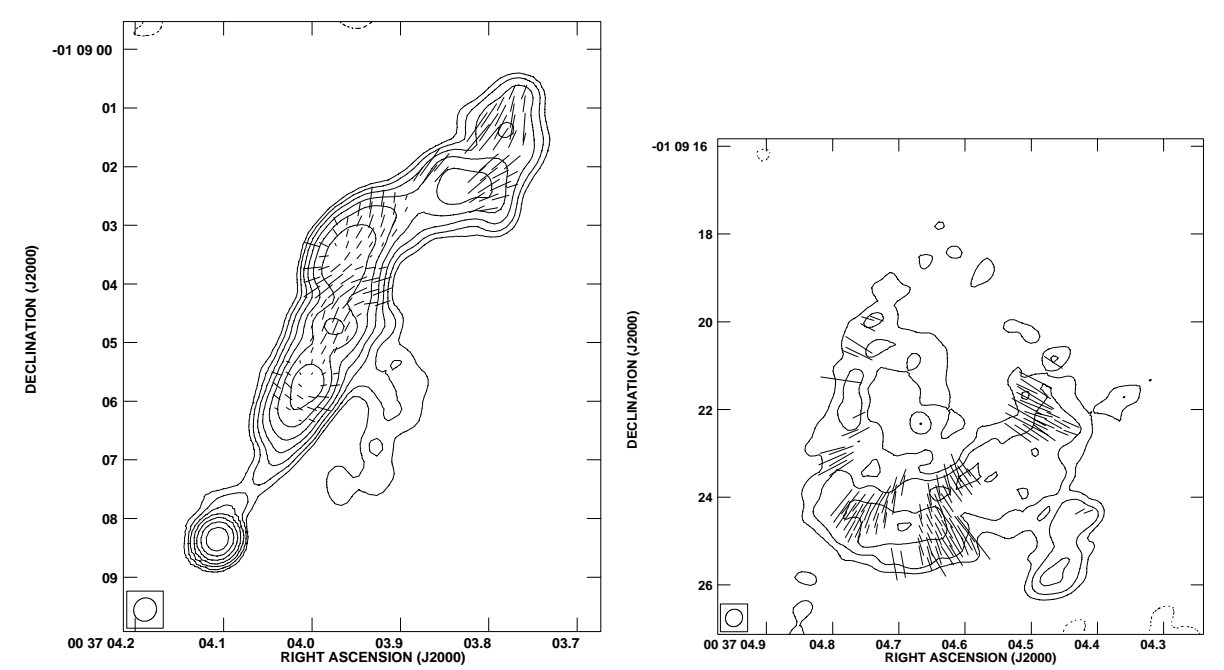

Fig. 16. Zoom-in of the jet (Right) and of the southern lobe (Left) of 0034-01 at 6-cm. with superimposed vectors indicating the projected electric field direction. The vectors are proportional in length to the fractional polarisation $(1$ arcsec $=1.0 \mathrm{ratio})$. The contour levels are: $0.25 \times-1,1,2,4,8,16,32,64,128 \mathrm{mJy}_{\text {beam }}^{-1}$

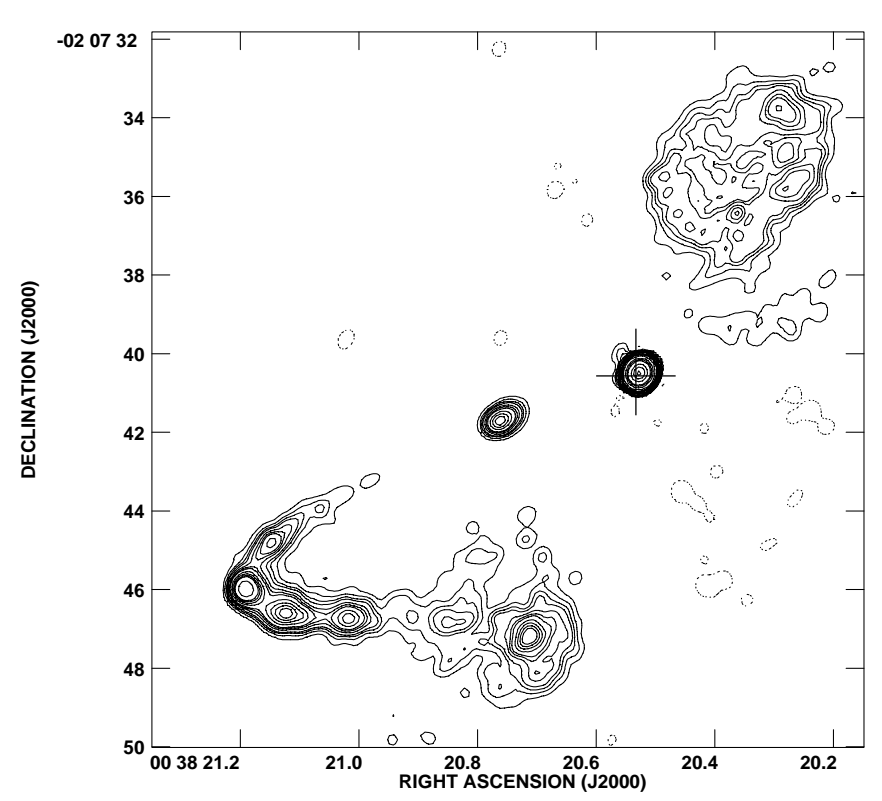

Fig. 17. Image of $0035-02$ at $6-\mathrm{cm}$. The contour levels are: $0.6 \times-1,1,2,3,4,6,8,10,15,20,25,30,50,100,150$, $200,400,600,800 \mathrm{mJy}^{\mathrm{m}} \mathrm{beam}^{-1}$. The peak flux is $514.6 \mathrm{mJy}$ beam $^{-1}$. The cross indicates the position of the optical galaxy

small (i.e. $\lesssim 70 \mathrm{kpc}$ ) radio sources. No obvious correlation has been found between the depolarization and the $R M$ (but see later for 0409-75).

If the depolarisation is produced by an external Faraday screen (as generally believed), this screen can be due to either the $\mathrm{X}$-ray halo around the radio source or to gas associated with the radio galaxy itself. In the latter case, we do not have the resolution high enough (specially in the ATCA data) to investigate in detail the structure of DP and $R M$ in the sources.
On the other hand, if the depolarisation is due to the X-ray halo around the radio source we can estimate the Faraday dispersion $(\Delta)$ for our small sources from the formula given in Garrington et al. (1991): $D P=$ $\exp \left[-2 k^{2} \Delta^{2}\left(\lambda_{1}^{2}-\lambda_{2}^{4}\right) /(1+z)^{4}\right]$ where $D P$ is the depolarization and $k=0.81$ if we want to obtain $\Delta$ in units of $\mathrm{cm}^{-3} \mu \mathrm{G}$ pc. For our frequencies we derive $\Delta=270(1+z)^{2}(-\ln D P)^{1 / 2}$ and we find values of the Faraday dispersion ranging from $\sim 800 \mathrm{~cm}^{-3} \mu \mathrm{G}$ pc for the E lobe of $0409-75$ to $\sim 240 \mathrm{~cm}^{-3} \mu \mathrm{G}$ pc for the E lobe of 0039-44. These values are typically higher than what found for extended sources (Garrington et al. 1991; Morganti et al. 1997b) but they lie on the established trend found by Garrington \& Conway (1991) between the linear size and the Faraday dispersion as do the CSS (Garrington \& Akujor 1996). For the CSS, this result is expected if they represent simply young version of the extended radio galaxies but living in a similar kind of environment. In this case, the trend between size and $\Delta$ would represent the radial decline of the density of the environment and the small radio sources in our sample could be, to first order, seen as an evolved phase of CSS on their way to becoming extended objects.

Of the three objects considered above, the only case in which both a large depolarization and a large $R M$ has been observed is 0409-75. It is interesting to note that this object also shows a quite low ionization state (e.g. [O III] $\lambda 5007 /[\mathrm{O} \mathrm{II}] \lambda 3727=0.19$, Dickson 1997) for a galaxy of such a high radio power. Thus, in the case of this object the weakness of [O III] $\lambda 5007$ could be due to a low ionization parameter, for instance, due to an interaction between the radio jet and a particularly rich ambient gas. 

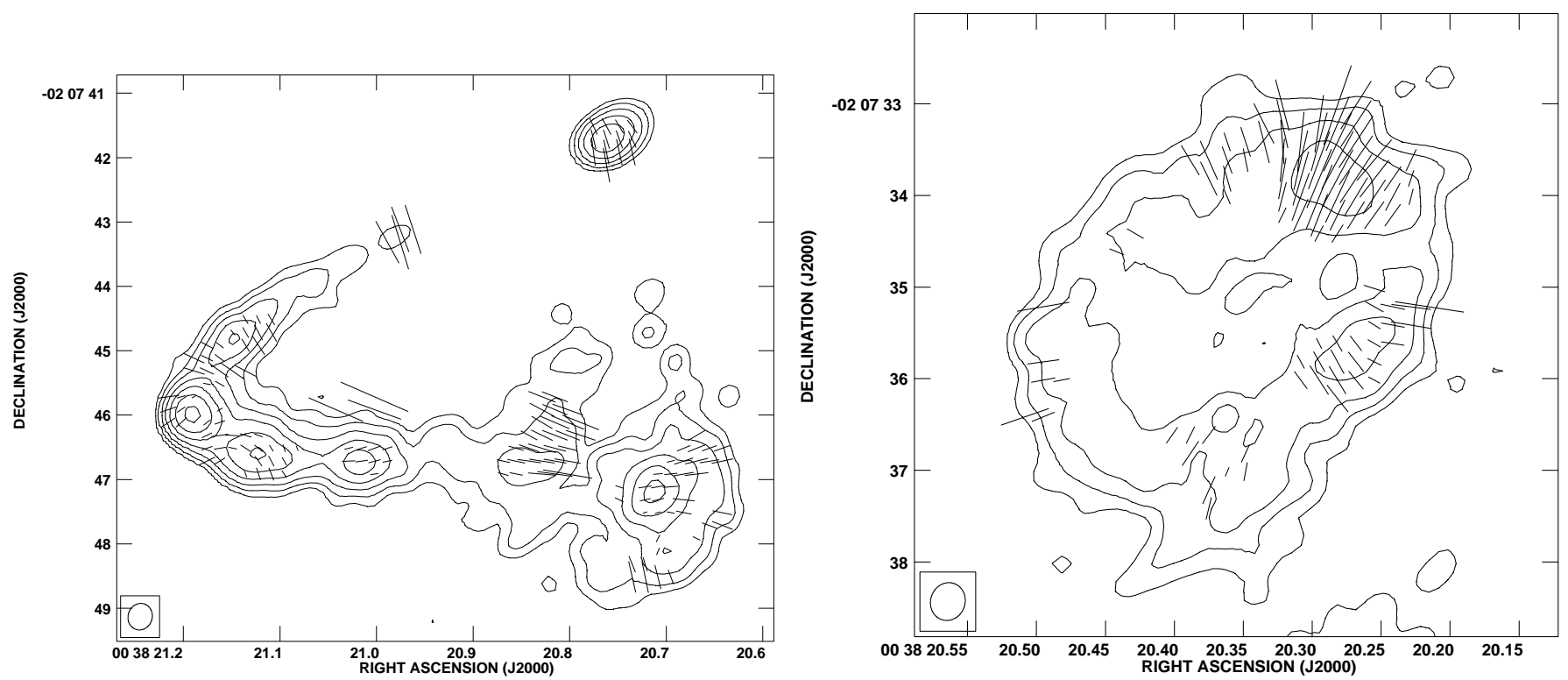

Fig. 18. Image of $0035-02$ at 6 -cm with superimposed vectors indicating the projected electric field direction. The vectors are proportional in length to the fractional polarisation $(1 \operatorname{arcsec}=1.0$ ratio). The contour levels are: $0.6 \times-1,1,2,4,8,16,32$,

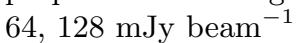

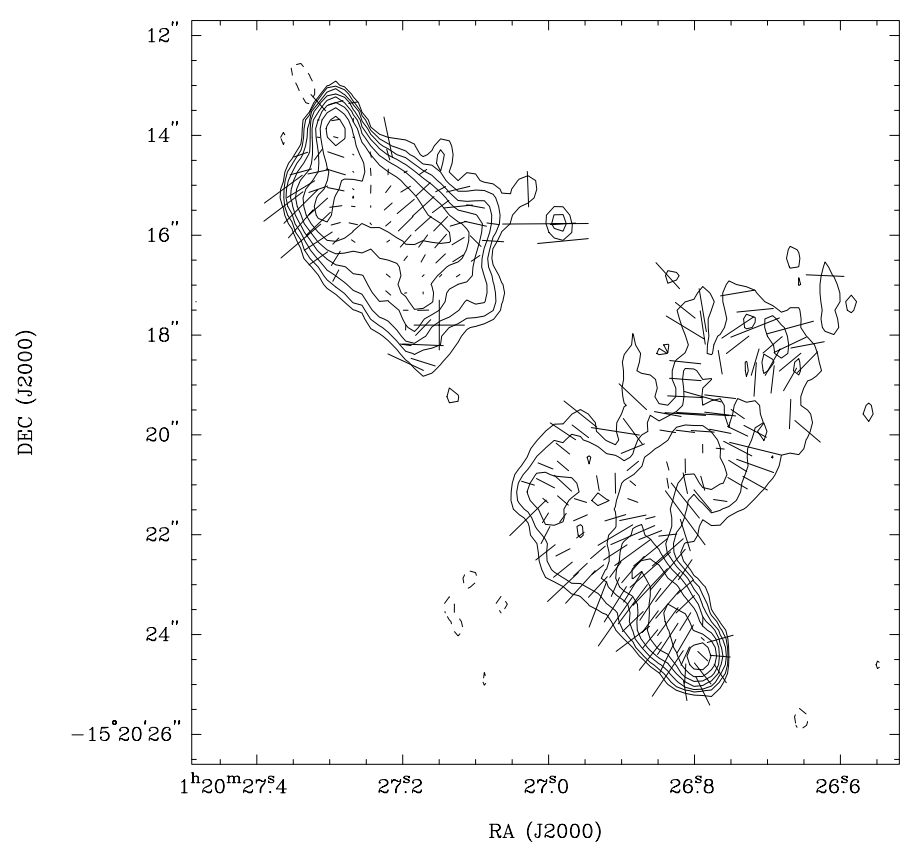

Fig. 19. Image of $0117-15$ at 6 -cm with superimposed vectors indicating the projected electric field direction. The vectors are proportional in length to the fractional polarisation $(1 \operatorname{arcsec}=0.081$ ratio). The contour levels are: $0.5 \times-1,1,2,4,8,16,32$, $64,128 \mathrm{mJy}_{\text {beam }}^{-1}$. The peak flux is $97.0 \mathrm{mJy} \mathrm{beam}^{-1}$. The cross indicates the position (from di Serego et al. 1994) of the optical galaxy 


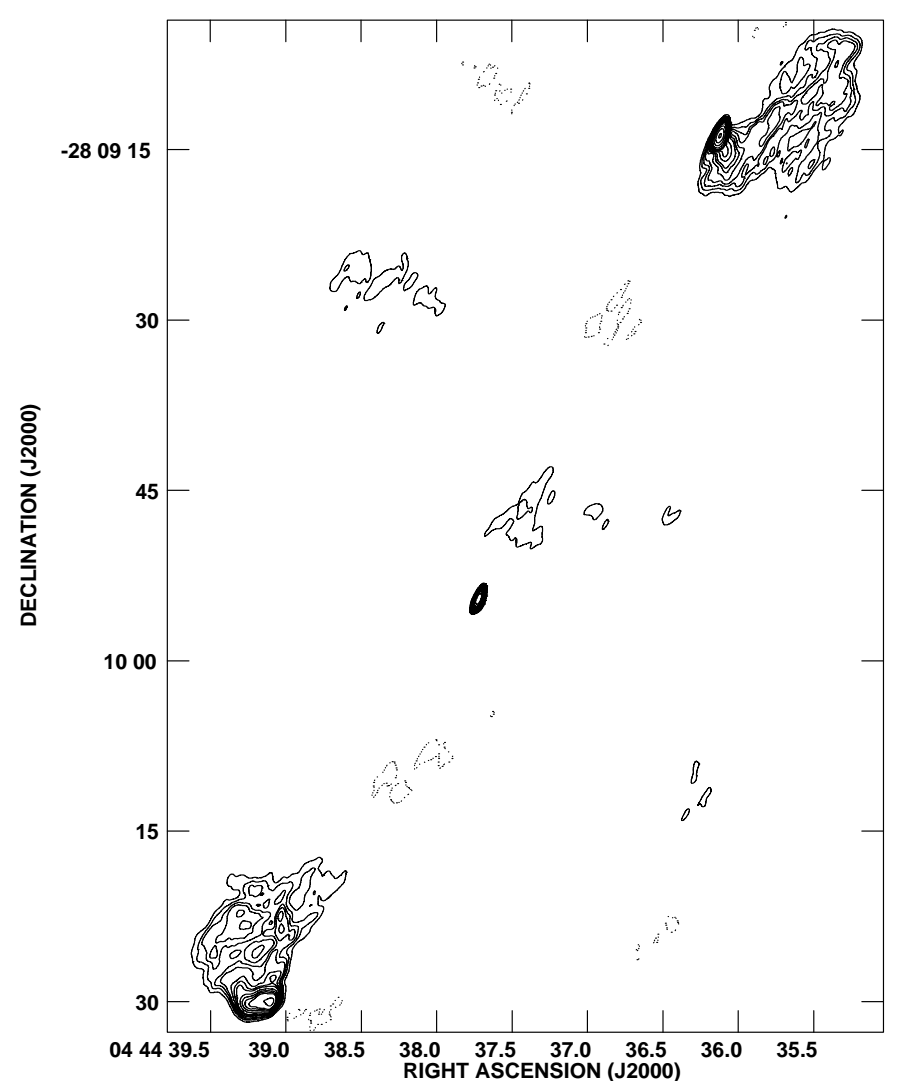

Fig. 20. Image of $0442-28$ at 6 -cm. The contour levels are: $0.45 \times-1,1,2,3,4,6,8,10,15,20,25,30,50,100,200,400 \mathrm{mJy}$ beam $^{-1}$. The peak flux is 197.0 mJy beam ${ }^{-1}$
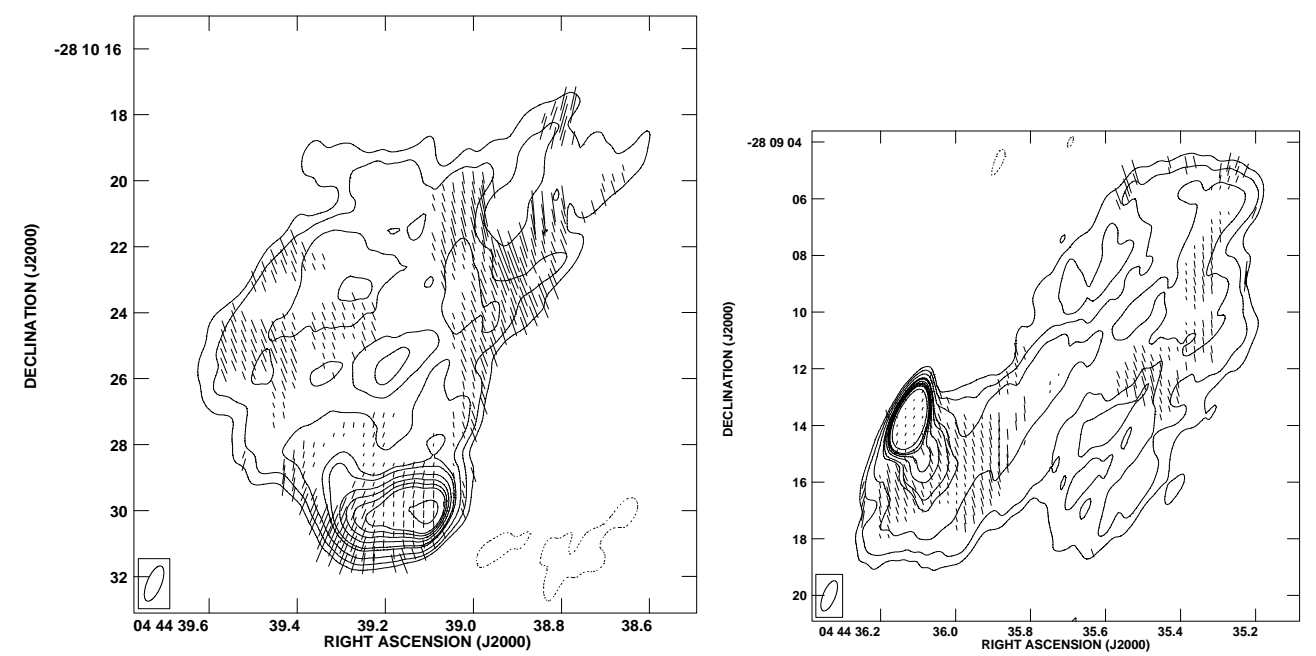

Fig. 21. Zoom-in of the southern (Right) and of the northern lobe (Left) of 0442-28 at 6-cm. with superimposed vectors indicating the projected electric field direction. The vectors are proportional in length to the fractional polarisation $(1$ arcsec $=1.0$ ratio). The contour levels are: $0.45 \times-1,1,2,4,8,10,15,20,25,30,40,50 \mathrm{mJy}^{\text {beam }}{ }^{-1}$ 


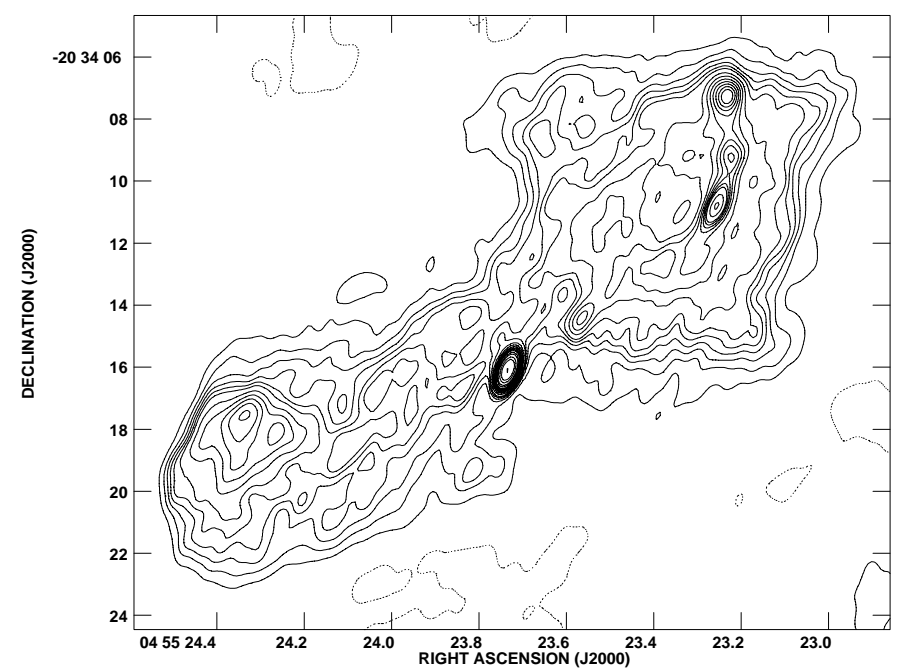

Fig. 22. Image of $0453-20$ at 6 -cm. The contour levels are: $0.40 \times-1,1,2,3,4,5,6,8,10,12,14,16,18,20,25,30,40,50$, $75 \mathrm{mJy}$ beam $^{-1}$. The peak flux is $30.4 \mathrm{mJy}_{\text {beam }^{-1}}$

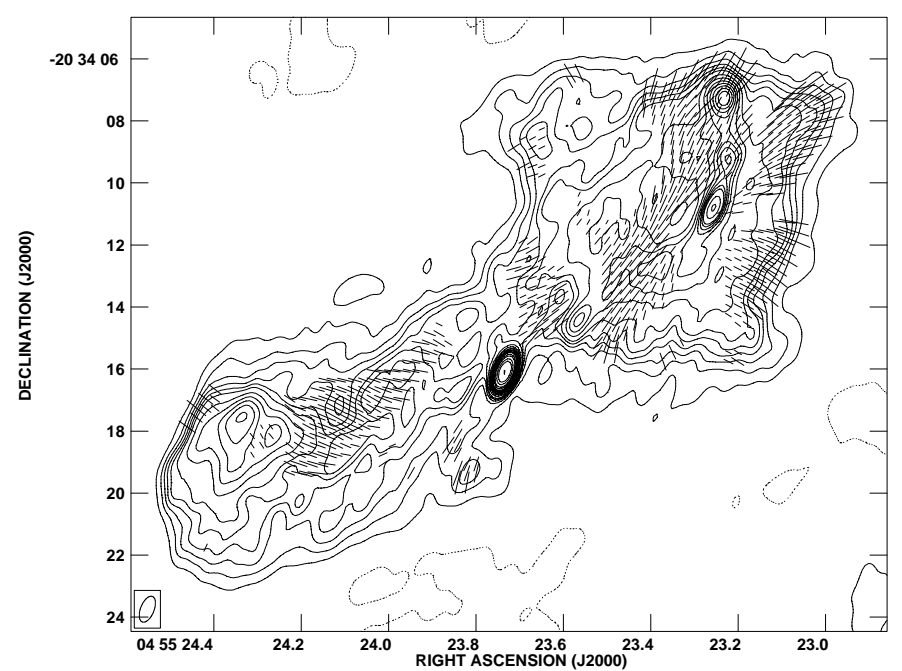

Fig. 23. Image of $0453-20$ at 6 -cm with superimposed vectors indicating the projected electric field direction. The vectors are proportional in length to the fractional polarisation $(1 \operatorname{arcsec}=1.7$ ratio). The contour levels are: $0.5 \times-1,1,2,3,4,5,6,8$, $10,12,14,16,18,20,25,30,40,50,75,100 \mathrm{mJy}_{\text {beam }^{-1}}$

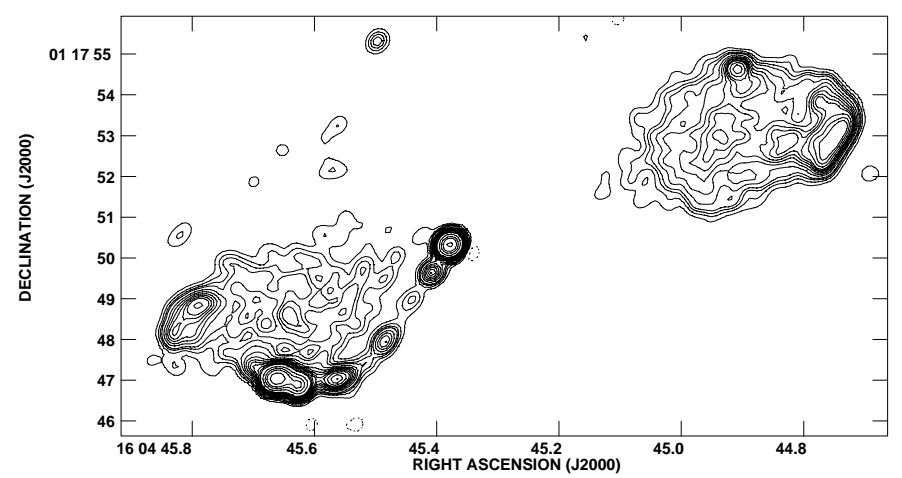

Fig. 24. Image of $1602+01$ at 6 -cm. The contour levels are: $0.45 \times-1,1,2,3,4,6,8,10,12,14,16,20,25,30,40,50,75,100$, 125 mJy beam $^{-1}$. The peak flux is $94.5 \mathrm{mJy}^{\text {beam }}{ }^{-1}$ 

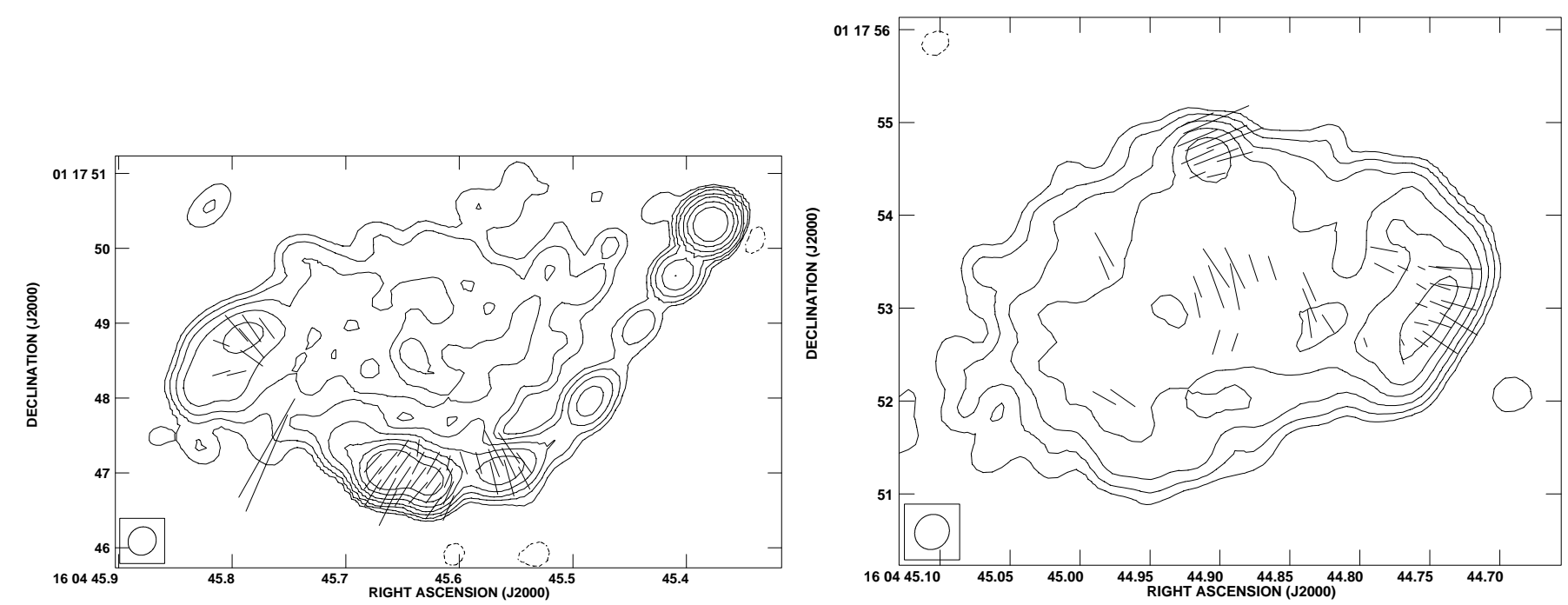

Fig. 25. Image of $1602+01$ at 6 -cm with superimposed vectors indicating the projected electric field direction. The vectors are proportional in length to the fractional polarisation $(1 \operatorname{arcsec}=0.5$ ratio). The contour levels are: $0.45 \times-1,1,2,4,8,16,32$, $64 \mathrm{mJy}$ beam $^{-1}$. The peak flux is $94.5 \mathrm{mJy}^{\text {beam }}{ }^{-1}$

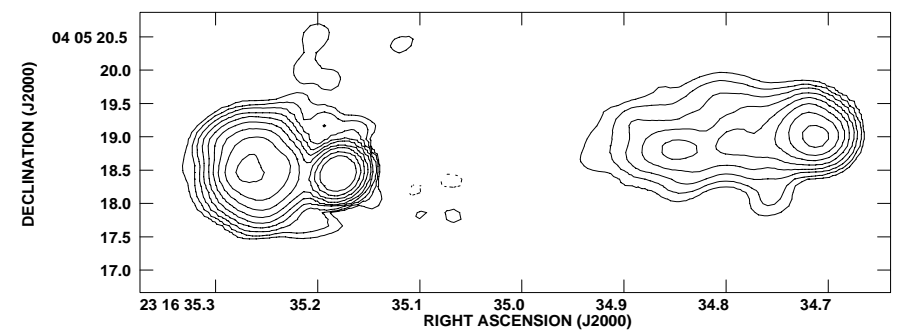

Fig. 26. Image of $2314+03$ at 6 -cm. The contour levels are: $0.45 \times-1,1,2,4,8,16,32,64,128,256$ mJy beam $^{-1}$. The peak flux is $400.7 \mathrm{mJy}^{-1}$
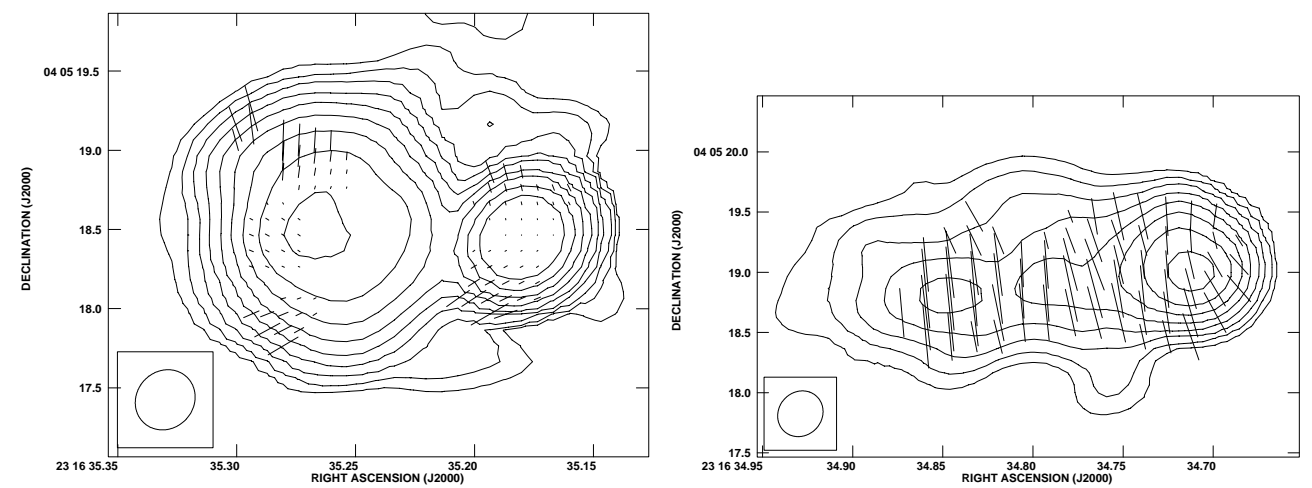

Fig. 27. Zoom-in of the eastern (Left) and of the western lobe (Right) of $2314+03$ at 6 -cm with superimposed vectors indicating the projected electric field direction. The vectors are proportional in length to the fractional polarisation $(1 \mathrm{arcsec}=0.25 \mathrm{ratio}$ for the eastern lobe and $1 \operatorname{arcsec}=0.5$ ratio for the western lobe). The contour levels are: $0.45 \times-1,1,2,4,8,16,32,64,128$, $256,512 \mathrm{mJy}^{\text {beam }^{-1}}$ 


\section{References}

Alvarez H., Aparici J., May J., Navarrete M., 1993, A\&A 271, 435

Baars, et al., 1977, A\&A 61, 99

Baum S.A., Heckman T., Bridle A.H., van Breugel W., Miley G., 1988, ApJS 68, 643

Christiansen W.N., Frater R.H., Watkinson A., O'Sullivan J.D., Lockhart I.A., Goss W.M., 1977, MNRAS 181, 183

Dickson, 1997, Ph.D. Thesis, University of Sheffield

Duncan \& Sproats 1992, PASA 10, 16

Ekers R.D., Fanti R., Lari C., Parma P., 1978, Nat 276, 588

Fanaroff B.L., Riley J.M., 1974, MNRAS 167, 31p

Fanti C., Fanti R., Dallacasa D., Schilizzi R.T., Spencer R.E., Stanghellini C., 1995, A\&A 302, 317

Laing R.A., Jenkins C.R., Wall J.V., Unger S.W., 1994, in "The Physics of Active Galaxies", Bicknell G.V., Dopita M.A., Quinn P.J. (eds.), ASP Conf. Ser. 54, 227

Laing R.A., 1996, in "Energy Transport in Radio Galaxies and Quasars", Hardee P.E., Bridle A.H., Zensus J.A. (eds.), ASP Conf. Ser. 100, 241

Leahy J.P., Williams A.G., 1984, MNRAS 210, 929

Leahy J.P., et al., 1997, MNRAS 291, 20

Lilly S.J., Prestage R.M., 1987, MNRAS 225, 531

Garrington S.T., Conway R.G., Leahy J.P., 1991, MNRAS 250, 171

Garrington S.T., Conway R.G., 1991, MNRAS 250, 198

Garrington S.T., Akujor C.E., 1996, in "Extragalactic radio sources", Ekers R., Fanti C., Padrielli L. (eds.). Kluwer Academic Publishers, p. 77

Gregorini L., de Ruiter H.R., Parma P., Sadler E.M., Vettolani G., Ekers R.D., 1994, A\&AS 106, 1

Hardcastle M.J., Alexander P., Pooley G.G., Riley J.M., 1998, MNRAS 296, 445

Hes R., 1995, Ph.D. Thesis, University of Groningen

Jones P.A., McAdam W.B., 1992, ApJS 80, 137
Morganti R., Killeen N.E.B., Tadhunter C.N., 1993, MNRAS 263, 1023

Morganti R., Oosterloo T.A., Fosbury R.A.E., Tadhunter C.N., 1995, MNRAS 274, 393

Morganti R., Oosterloo T.A., Reynolds J., Tadhunter C.N., Migenes V., 1997a, MNRAS 284, 541

Morganti R., Tadhunter C.N., 1997, Mem. S.A.It. 68, 243

Morganti R., Parma P., Capetti A., Fanti R., de Ruiter H.R., Prandoni I., 1997b, A\&AS 126, 335

Padovani P., Morganti R., Siebert J., Cimatti A., Vagnetti F., 1999, MNRAS 304, 829

Prestage R.M., Peacock J.A., 1983, MNRAS 204, 355

Reynolds J.E., 1996, in: "Australia Telescope Compact Array, Users's Guide", Walsh W.M. \& McKay D.J. (eds.)

Sault R.J., Teuben P.J., Wright M.C.H., 1995, in "Astronomical Data Analysis Software and Systems IV" Shaw R., Payne H.E. and Hayes J.J.E. (eds.), Astron. Soc. Pac. Conf. Ser. 77, 433

di Serego Alighieri S., Danziger J., Morganti R., Tadhunter C.N., 1994, MNRAS 269, 998

Siebert J., Brinkmann W., Morganti R., Tadhunter C., Danziger J., Fosbury R., di Serego Alighieri, 1996, MNRAS 279, 1331

Tadhunter C.N., Morganti R., di Serego Alighieri S., Fosbury R.A.E., Danziger I.J., 1993, MNRAS 263, 999

Tadhunter C.N., Dickson R., Morganti R., Villar-Martin M., 1997, in proceeding of ESO/IAC workshop "Quasar Hosts", Clements \& Perez-Fournon (eds.), p. 311

Tadhunter C.N., Morganti R., Robinson A., Dickson R., VillarMartin M., Fosbury R.A.E., 1998, MNRAS 298, 1035

Trussoni E., Vagnetti F., Massaglia S., Feretti L., Parma P., Morganti R., Fanti R., Padovani P., 1999, A\&A 348, 437

Ulvestad J.S., 1985, ApJ 288, 514

Venturi T., Morganti R., Tzioumis A., et al., 1996, 3rd AsiaPacific Telescope Workshop, King E. (ed.), p. 287

Wall J.V., Peacock J.A., 1985, MNRAS 216, 173 